UCSB Math 2013-05

NSF-KITP-13-076

\title{
New Methods for Characterizing Phases of 2D Supersymmetric Gauge Theories
}

\author{
James Halverson ${ }^{1}$, Vijay Kumar ${ }^{1}$, and David R. Morrison ${ }^{2,3}$ \\ ${ }^{1}$ Kavli Institute for Theoretical Physics, University of California \\ Santa Barbara, CA 93106, USA \\ ${ }^{2}$ Department of Mathematics, University of California \\ Santa Barbara, CA 93106, USA \\ ${ }^{3}$ Department of Physics, University of California \\ Santa Barbara, CA 93106, USA \\ jim@kitp.ucsb.edu, vijayk@kitp.ucsb.edu, drm@math.ucsb.edu
}

\begin{abstract}
We study the physics of two-dimensional $\mathcal{N}=(2,2)$ gauged linear sigma models (GLSMs) via the two-sphere partition function. We show that the classical phase boundaries separating distinct GLSM phases, which are described by the secondary fan construction for abelian GLSMs, are completely encoded in the analytic structure of the partition function. The partition function of a non-abelian GLSM can be obtained as a limit from an abelian theory; we utilize this fact to show that the phases of non-abelian GLSMs can be obtained from the secondary fan of the associated abelian GLSM. We prove that the partition function of any abelian GLSM satisfies a set of linear differential equations; these reduce to the familiar $A$-hypergeometric system of Gel'fand, Kapranov, and Zelevinski for GLSMs describing complete intersections in toric varieties. We develop a set of conditions that are necessary for a GLSM phase to admit an interpretation as the low-energy limit of a non-linear sigma model with a Calabi-Yau threefold target space. Through the application of these criteria we discover a class of GLSMs with novel geometric phases corresponding to Calabi-Yau manifolds that are branched double-covers of Fano threefolds. These criteria provide a promising approach for constructing new Calabi-Yau geometries.
\end{abstract}

September 10, 2018 


\section{Introduction}

The gauged linear sigma model (GLSM) has provided many insights on the space of Calabi-Yau threefolds [1]. It is a two-dimensional supersymmetric gauge theory with charged matter fields which, if the degrees are constrained correctly, flows to a supersymmetric conformal field theory (SCFT) at low energies. For a large class of Calabi-Yau threefolds $X$, with a judicious choice of gauge group, matter content and interactions one can design a GLSM that flows to the same SCFT as a non-linear sigma model (NLSM) whose target space is $X$. Various geometric properties of $X$, such as its quantum moduli space (the space of marginal deformations of the SCFT), can be analyzed in terms of GLSM physics. For example, the properties of distinct classical phases in the GLSM determine the nature of singular points in the (compactified) moduli space [1].

Recently, it was conjectured that the two-sphere partition function of a GLSM - which was computed independently in [2, 3] - allows one to determine the Gromov-Witten invariants of Calabi-Yau threefolds [4]. A compelling physical argument for this conjecture has been given in [5]. For Calabi-Yau threefold\$1 that can be modeled with a GLSM, this new approach allows one to compute these invariants and the exact metric on moduli space without the use of mirror symmetry [4,7,8].

Given these previous successes, in this paper we use the two-sphere partition function as a lens for studying GLSM physics. We focus our attention on three problems: defining the (classical) boundaries separating distinct GLSM phases, determining whether a given GLSM phase is geometric, and defining a system of differential equations on the moduli space of the low-energy SCFT.

The partition function takes the form of a contour integral for both abelian and non-abelian GLSMs. We show that as far as the partition function is concerned, the physics of the nonabelian theory can be extracted from an abelian theory that we call the associated Cartan theory. This proposal resonates with an early proposal made by Hori and Vafa [9], is related to some developments in the mathematical study of Gromov-Witten invariants [10, and also appeared more recently in the context of the partition function [2. The analysis of the physics of nonabelian GLSMs can be quite involved, as discussed in [11] for example. We utilize the associated Cartan theory to extend many of our results for abelian theories to non-abelian theories.

We demonstrate that classical phase boundaries in the GLSM are encoded in the analytic structure of the partition function integrand. We show that the boundaries as determined by the two-sphere partition function reproduce the known results (the structure of the secondary fan) for abelian GLSMs. Moreover, we show that the phase boundaries for non-abelian GLSMs can be obtained from the secondary fan of the associated Cartan theory.

We show that the partition function can be used as a tool to determine whether a given phase in a GLSM is geometric, i.e., whether the low-energy physics is approximated by an NLSM with a smooth Calabi-Yau threefold as its target space. This question has traditionally been answered by a study of the solutions to the D-term and F-term equations [1,12, which can be non-trivial as illustrated by the Hori-Tong analysis of the GLSM for Rødland's Pfaffian Calabi-Yau threefold [11]. We formulate a set of conditions, which we refer to as the geometric phase criteria, that are necessary for a given GLSM phase to be geometric. These conditions provide a valuable cross check when the D-term and F-term analysis is subtle, as was the case in [11].

\footnotetext{
${ }^{1}$ There has also been an extension to Calabi-Yau fourfolds [6].
} 
The GLSM, together with the geometric phase criteria, can be a valuable tool to explore the space of Calabi-Yau threefolds. Rather than starting with a known Calabi-Yau threefold $X$ and then constructing a GLSM that models $X$ in one of its phases, we start with a general collection of GLSMs and focus our attention on the subcollection of GLSMs which satisfy the geometric phase criteria. Apart from reproducing the list of known Calabi-Yau threefolds, a closer analysis of this subcollection could potentially lead to new constructions of Calabi-Yau threefolds.

We provide an instantiation of this approach by considering a collection of GLSMs with gauge group $U(1)$. We find a twenty member subcollection that satisfy the geometric phase criteria. Five of these examples correspond to known Calabi-Yau threefolds that are complete intersections in projective space. A closer analysis of the remaining fifteen examples suggests that the phase in which the geometric criteria are satisfied is a so-called "hybrid phase." We then provide a physical argument which shows that these fifteen examples are actually geometric and correspond to doublecovers of Fano threefolds branched over a surface of appropriate degree. These examples are close relatives of a phase studied in [13, 14] that occurs in a GLSM for a complete intersection of four quadrics in $\mathbb{P}^{7}$. This GLSM phase was argued in those papers to correspond to a Calabi-Yau threefold geometry (a non-commutative resolution of a double-cover of $\mathbb{P}^{3}$ branched over a singular octic surface) 2 The examples that we uncover are simpler, more traditional smooth Calabi-Yau geometries that appear as hybrid phases in a GLSM.

The moduli space of an $N=2$ SCFT has a rich structure that is known as special geometry [16 22. Such a moduli space of dimension $s$ carries an $S p(2 s+2, \mathbb{Z})$ bundle to which we can associate a set of differential equations [23 25] with regular singular points [26]. The Picard-Fuchs differential equations that describe the variation of complex structure of Calabi-Yau threefolds are an example. For threefolds that arise as complete intersections in toric varieties, the solutions to the PicardFuchs equations are annihilated [27 $[29]$ by the differential operators of the $A$-hypergeometric system [30, 31] of Gel'fand, Kapranov, and Zelevinski (GKZ). We show that one can associate a set of differential equations, which we call the $A$-system, to any GLSM with an abelian gauge group. The $A$-system is a slight generalization of the $A$-hypergeometric system and applies to a larger class of $N=2$ SCFTs.

The outline of the paper is as follows: section 2 discusses the analytic structure of the twosphere partition function and introduces the associated Cartan theory; section 3 is a discussion of GLSM phases from the partition function point of view; section 4 introduces the geometric phase criteria, which are then applied in section 5 to a collection of GLSMs to discover novel Calabi-Yau threefold geometries; section 6 shows that we can associate a system of differential equations to the moduli space of any abelian GLSM; we summarize our findings and discuss possible future directions in section 7 .

\section{Two-Sphere Partition Function}

In this section we will review the two-sphere partition function of $d=2 \mathcal{N}=(2,2)$ GLSMs and discuss aspects of its structure that will be useful in sections 3 and 4 . We also introduce the necessary notation that will be used in the rest of the paper.

We consider an $\mathcal{N}=(2,2)$ GLSM with gauge group $G$, where $G$ is a compact Lie group with associated Lie algebra $\mathfrak{g}$, and $n$ chiral multiplets $\Phi_{i=1, \cdots, n}$ in irreducible representations $R_{i}$ of $G$.

\footnotetext{
${ }^{2}$ See also the recent work that utilizes the two-sphere partition function to argue for such a geometric interpretation [15].
} 
By an abuse of notation we will also use $R_{i}$ to denote the representation of the corresponding Lie algebra $\mathfrak{g}$. We require the existence of a $U(1)_{V}$ R-symmetry and denote the R-charge of $\Phi_{i}$ by $\mathrm{q}_{i}$. Let $\mathfrak{t}_{\mu}, \mu=1, \cdots, \operatorname{rank}(\mathfrak{g})$, denote the generators of the Cartan subalgebra. We will assume that a non-empty subset of these generators, $\left\{\mathfrak{t}_{1}, \cdots, \mathfrak{t}_{s}\right\}$, commutes with every element of the Lie algebra $\mathfrak{g}$. Without loss of generality we have assumed these to be the first $s$ generators. For each $\mathfrak{t}_{a=1, \cdots, s}$ we have a Fayet-Iliopoulos (FI) parameter $r_{a}$ and a periodic theta angle $\theta_{a}$, which we write using vector notation as $\boldsymbol{r} \in \mathbb{R}^{s}$ and $\boldsymbol{\theta} \in(\mathbb{R} /(2 \pi \mathbb{Z}))^{s}$. These parameters can be naturally combined as $z_{a}=\exp \left(-2 \pi r_{a}+i \theta_{a}\right) \in \mathbb{C}^{*}$. Throughout the paper $s$ will denote the number of FI parameters with the index $a=1, \cdots, s$, and the index $\mu=1, \cdots, \operatorname{rank}(\mathfrak{g})$.

We will consider GLSMs that flow to superconformal field theories (SCFTs) at low energies. A necessary condition for this is that the axial $U(1)$ R-symmetry be non-anomalous [1]: $\sum_{i=1}^{n} \operatorname{tr}_{R_{i}}(\mathfrak{t})=$ 0 , for all $\mathfrak{t} \in \mathfrak{g}$. The central charge of the resulting SCFT is given by [32]: $\hat{c}=c / 3=\sum_{i=1}^{n}(1-$ $\left.\mathbf{q}_{i}\right) \operatorname{dim}\left(R_{i}\right)-\operatorname{dim}(\mathfrak{g})$.

The two-sphere partition function in the Coulomb branch representation is given by [2, 3 ]

$$
Z_{S^{2}}\left(z_{a}, \bar{z}_{a}\right)=\frac{1}{|\mathcal{W}|} \sum_{m \in \mathbb{Z}^{\operatorname{rank}(\mathfrak{g})}} \int\left(\prod_{\mu=1}^{\operatorname{rank}(\mathfrak{g})} \frac{d \tau_{\mu}}{2 \pi i}\right) Z_{\text {class }} Z_{\text {gauge }} \prod_{i} Z_{\phi_{i}}
$$

where the classical factor $Z_{\text {class }}$ and the one-loop determinants $Z_{\phi_{i}}$ and $Z_{\text {gauge }}$ are given by

$$
\begin{aligned}
Z_{\text {class }} & =e^{-4 \pi \boldsymbol{r} \cdot \boldsymbol{\tau}-i \boldsymbol{\theta} \cdot \boldsymbol{m}}=\prod_{a=1}^{s}\left(z_{a}\right)^{\tau_{a}-m_{a} / 2}\left(\bar{z}_{a}\right)^{\tau_{a}+m_{a} / 2} \\
Z_{\text {gauge }} & =\prod_{\alpha \in \mathcal{P}}\left(\frac{\left(\alpha_{\mu} m_{\mu}\right)^{2}}{4}-\left(\alpha_{\mu} \tau_{\mu}\right)^{2}\right) \\
Z_{\phi_{i}} & =\prod_{\rho^{i} \in R_{i}} \frac{\Gamma\left(\frac{\mathbf{q}_{i}}{2}-\rho_{\mu}^{i}\left(\tau_{\mu}+\frac{m_{\mu}}{2}\right)\right)}{\Gamma\left(1-\frac{\mathrm{q}_{i}}{2}+\rho_{\mu}^{i}\left(\tau_{\mu}-\frac{m_{\mu}}{2}\right)\right)} .
\end{aligned}
$$

In the above formulae $\left(\alpha_{\mu}\right) \in \mathcal{P}$, the set of positive roots of $\mathfrak{g}$, and the set of $\left(\rho_{\mu}^{i}\right)$ denotes the weights of the representation $R_{i}$ of $\mathfrak{g}$. Note that $\alpha_{\mu}$ and $\rho_{\mu}^{i}$ are vectors in the weight lattice $\cong \mathbb{Z}^{\operatorname{rank}(\mathfrak{g})}$. $\mathcal{W}$ denotes the Weyl group of $\mathfrak{g}$ and $|\mathcal{W}|$, its cardinality. The boldface notation in (2.2) is used throughout the paper as a shorthand for $s$-tuples. The integration is performed over the imaginary axis for each $\tau_{\mu}$. When the R-charges $\mathrm{q}_{i}$ are all positive, which we will assume to be the case, there are no poles on the contour of integration. In some cases, we take a limit where the R-charges go to zero from the positive direction for simplicity.

Most of the discussion in this paper will focus on GLSMs where the gauge group $G$ is abelian. Note that $s=\operatorname{rank}(\mathfrak{g})=\operatorname{dim}(\mathfrak{g})$ in this case. We will denote the charges of the chiral fields by the vectors $\boldsymbol{Q}_{i} \in \mathbb{Z}^{s}, i=1, \cdots, n$. The partition function in this case reads

$$
Z_{S^{2}}\left(z_{a}, \bar{z}_{a}\right)=\sum_{\boldsymbol{m} \in \mathbb{Z}^{s}} \int\left(\prod_{a=1}^{s} \frac{d \tau_{a}}{2 \pi i}\left(z_{a}\right)^{\tau_{a}-m_{a} / 2}\left(\bar{z}_{a}\right)^{\tau_{a}+m_{a} / 2}\right) \prod_{i=1}^{n} \frac{\Gamma\left(\frac{\mathrm{q}_{i}}{2}-\boldsymbol{Q}_{i} \cdot\left(\boldsymbol{\tau}+\frac{\boldsymbol{m}}{2}\right)\right)}{\Gamma\left(1-\frac{\mathrm{q}_{i}}{2}+\boldsymbol{Q}_{i} \cdot\left(\boldsymbol{\tau}-\frac{\boldsymbol{m}}{2}\right)\right)}
$$

In a GLSM the complexified FI parameters, $z_{a}$, correspond to exact marginal parameters of the IR SCFT and parameterize a moduli space $\mathcal{M}$. When the SCFT has a geometric interpretation - as the low-energy limit of a non-linear sigma model (NLSM) with target space a Calabi-Yau 
threefold $X$ - the moduli space $\mathcal{M}$ corresponds to (part of) the quantum Kähler moduli space of $X 3$ It was conjectured in [4] that the two-sphere partition function computes the exact Kähler potential, and hence the exact Kähler metric, on $\mathcal{M}$. More precisely,

$$
Z_{S^{2}}=\exp \left(-K\left(z_{a}, \bar{z}_{a}\right)\right)
$$

where $K$ is the Kähler potential on $\mathcal{M}$. A compelling physical argument for this conjecture has been provided by Gomis and Lee [5].

\section{Non-abelian GLSMs and their associated Cartan Theory}

It is apparent from the structure of (2.1) that the partition function of a non-abelian GLSM is closely related to the partition function of an abelian GLSM. The maximal torus $\mathcal{T}_{G}$ of $G$ is the gauge group of the abelian theory, with Lie algebra equal to the Cartan subalgebra $\mathfrak{u}(1)^{\oplus \operatorname{rank}(\mathfrak{g})}$ of $G$. We will henceforth refer to this abelian theory as the associated Cartan theory. Each chiral multiplet $\Phi_{i}$ of the GLSM reduces to $\operatorname{dim}\left(R_{i}\right)$ chiral multiplets with charges specified by the weights of the representation - the vectors $\left(\rho^{i}\right) \in \mathbb{Z}^{\operatorname{rank}(\mathfrak{g})}$. Note that we can rewrite $Z_{\text {gauge }}$ as

$$
Z_{\text {gauge }}=\prod_{\alpha \in \mathcal{P}}\left(\frac{\left(\alpha_{\mu} m_{\mu}\right)^{2}}{4}-\left(\alpha_{\mu} \tau_{\mu}\right)^{2}\right)=\left(\prod_{\alpha \in \mathcal{P}} e^{i \pi \sum_{\mu} \alpha_{\mu} m_{\mu}}\right)\left(\prod_{\alpha \in \mathcal{R}} \frac{\Gamma\left(1-\alpha_{\mu}\left(\tau_{\mu}+\frac{m_{\mu}}{2}\right)\right.}{\Gamma\left(\alpha_{\mu}\left(\tau_{\mu}-\frac{m_{\mu}}{2}\right)\right)}\right),
$$

where $\mathcal{R}$ is the set of roots of $\mathfrak{g}$. This leads us to include $\operatorname{dim}(\mathfrak{g})-\operatorname{rank}(\mathfrak{g})$ additional chiral multiplets with charges given by the roots $\left(\alpha_{\mu}\right)$ and R-charge 2. The Cartan theory has a total of $\operatorname{rank}(\mathfrak{g})$ FI parameters with an additional $\operatorname{rank}(\mathfrak{g})-s$ parameters, denoted $\left(\tilde{z}_{\mu}\right), \mu=s+1, \cdots, \operatorname{rank}(\mathfrak{g})$, as compared to the original non-abelian theory. The partition functions of the two theories, by construction, are related as

$$
Z_{S^{2}}^{N A}\left(z_{a}, \bar{z}_{a}\right)=Z_{S^{2}}^{C}\left(z_{a}, \bar{z}_{a}, \tilde{z}_{\mu}=e^{2 \pi i \gamma_{\mu}}, \overline{\tilde{z}}_{\mu}=e^{-2 \pi i \gamma_{\mu}}\right)
$$

where $\gamma=\frac{1}{2} \sum_{\alpha \in \mathcal{P}} \alpha_{\mu}$ is the so-called Weyl vector. We use the labels $C$ and $N A$ to denote the Cartan theory and the original non-abelian theory respectively. We emphasize that the analytic structure of the $Z_{S^{2}}$ integrands are identical in these two theories; the partition functions differ only in the $Z_{\text {class }}$ factor according to the dependence on FI parameters.

The idea of associating an abelian GLSM to a non-abelian GLSM has been studied in the past starting with Hori and Vafa [9]. It is conceivable that the relationship between the Cartan theory and the non-abelian theory extends beyond the equality of their partition functions. For example, if we assume that both the non-abelian theory and the associated Cartan theory flow to SCFTs at low energies, the central charges are equal -

$$
\hat{c}_{C}=\sum_{i=1}^{n} \operatorname{dim}\left(R_{i}\right)\left(1-\mathrm{q}_{i}\right)+(\operatorname{dim}(\mathfrak{g})-\operatorname{rank}(\mathfrak{g}))(-1)-\operatorname{rank}(\mathfrak{g})=\hat{c}_{N A} .
$$

We leave the investigation of this relationship to future work.

\footnotetext{
${ }^{3}$ In general, $\mathcal{M}$ is a subset of the full Kähler moduli space, since extra exact marginal operators can be emergent at the IR fixed point.
} 


\section{Analytic structure of the integrand}

The integrand in equation (2.1) is a meromorphic function of the integration variables. We restrict ourselves to abelian gauge theories without loss of generality; when the GLSM is non-abelian, the analytic structure of the partition function is determined by the associated Cartan theory. The partition function for an abelian GLSM with $G=U(1)^{s}$ is

$$
Z_{S^{2}}=\int\left(\prod_{a=1}^{s} \frac{d \tau_{a}}{2 \pi i}\right) \sum_{\boldsymbol{m} \in \mathbb{Z}^{s}} e^{-4 \pi \boldsymbol{r} \cdot \boldsymbol{\tau}-i \boldsymbol{\theta} \cdot \boldsymbol{m}} \prod_{i=1}^{n} Z_{\phi_{i}},
$$

with

$$
Z_{\phi_{i}}=\frac{\Gamma\left(\frac{\mathrm{q}_{i}}{2}-\boldsymbol{Q}_{i} \cdot\left(\boldsymbol{\tau}+\frac{\boldsymbol{m}}{2}\right)\right)}{\Gamma\left(1-\frac{\mathrm{q}_{i}}{2}+\boldsymbol{Q}_{i} \cdot\left(\boldsymbol{\tau}-\frac{\boldsymbol{m}}{2}\right)\right)} .
$$

Recall that $\Gamma(z)$ is a meromorphic function in the complex plane with simple poles at $z=-n$, $n \in \mathbb{Z}_{\geq 0}$, with residue $\operatorname{Res}_{z=-n} \Gamma(z)=\frac{(-1)^{n}}{n !}$ and an essential singularity at $z=\infty$. Each factor $Z_{\phi_{i}}$ in the integrand has poles along the hyperplanes

$$
H_{i}^{(k)}: \quad \frac{\mathrm{q}_{i}}{2}-\boldsymbol{Q}_{i} \cdot\left(\boldsymbol{\tau}+\frac{\boldsymbol{m}}{2}\right)=-k, \quad k \in \mathbb{Z}_{\geq 0}, \quad k \geq \boldsymbol{Q}_{i} \cdot \boldsymbol{m} .
$$

The partition function integrand can be regarded as a meromorphic function on the space $\mathbb{C}^{\text {rank(g) }}$ with poles along the hyperplanes $H_{i}^{(k)}$, which we refer to as polar divisors. Similarly, each factor $Z_{\phi_{i}}$ has a zero along the hyperplanes

$$
\mathfrak{H}_{i}^{(k)}: \quad 1-\frac{\mathrm{q}_{i}}{2}+\boldsymbol{Q}_{i} \cdot\left(\boldsymbol{\tau}-\frac{\boldsymbol{m}}{2}\right)=-k, \quad k \in \mathbb{Z}_{\geq 0}, \quad k \geq \boldsymbol{Q}_{i} \cdot \boldsymbol{m},
$$

which we refer to as zero divisors. Note that the collection of hyperplanes $H_{i}^{(k)}$ is contained in the half space $\boldsymbol{Q}_{i} \cdot \boldsymbol{\tau} \geq 0$, while the $\mathfrak{H}_{i}^{(k)}$ are contained in the half space $\boldsymbol{Q}_{i} \cdot \boldsymbol{\tau} \leq 0$. The analytic structure of the integrand will be relevant in what follows, as we will evaluate the integral in (2.9) through the method of residues.

\section{Phases of Gauged Linear Sigma Models}

The low-energy effective theory describing the dynamics of the GLSM depends on the value of the FI parameters [1]. The space of FI parameters can be divided into phase regions depending on the character of the low-energy dynamics, and the analysis of the phase structure of a GLSM typically proceeds through a close study of the D-terms and F-terms. In this section we discuss how this information can be easily extracted from $Z_{S^{2}}$.

The idea is stated rather simply: when $Z_{S^{2}}$ is evaluated by the method of residues, the contour prescription depends on the value of the FI parameters, which in turn affects the set of poles that contribute to the integral. At certain codimension-one walls in FI parameter space the structure of poles contributing to the $Z_{S^{2}}$ integral can change, signaling the presence of a GLSM phase transition along that wall. In particular, for abelian GLSMs we show that this recovers the description of phases in terms of the "secondary fan" [33 35]. Furthermore, we also demonstrate that phases of 
non-abelian GLSMs can be understood in terms of phases of the associated Cartan theory.

\subsection{Evaluation of the Partition Function}

We use the multi-dimensional residue method (see [36], for example) to evaluate the $s$-dimensional integral in (2.9) for an abelian GLSM as defined in section 2, Let $I$ denote an $s$-element subset of $\{1, \cdots, n\}$ such that the $s$ vectors $\left\{\boldsymbol{Q}_{i \in I}\right\}$ are linearly independent, and let $\mathfrak{I}$ denote the set of all such subsets $I$. The corresponding polar divisors $H_{i \in I}^{(k)}$ for all $k \in \mathbb{Z}_{\geq 0}$ (defined in (2.11) ), intersect in an infinite discrete point set that we denote by $P_{I}$. The residue of the integrand at any point $p \in P_{I}$ is well defined and can be evaluated. $Z_{S^{2}}$ is a sum of the residues at all points $p \in \bigcup_{I \in \mathfrak{I}} P_{I}$ that lie inside the domain of integration.

The integration in (2.9) is carried out over the imaginary axis from $-i \infty$ to $+i \infty$ for each $\tau_{a}$. Each of the $s$ contours can be closed at infinity based on the asymptotic behavior of the integrand, which is entirely determined by the exponential factor, $e^{-4 \pi \boldsymbol{r} \cdot \boldsymbol{\tau}}$, for sufficiently large $|\boldsymbol{r}| \underline{4}$ Given a set $I$ as defined above, we express the FI parameters as $\boldsymbol{r}=\sum_{i \in I} r_{i} \boldsymbol{Q}_{i}$. We have

$$
Z_{\text {class }} \sim e^{-4 \pi r_{i} \boldsymbol{Q}_{i} \cdot \tau}
$$

If $r_{i}>0$, for all $i \in I$, the points $P_{I}$ lie inside the domain of integration when the contours are closed at infinity, and $Z_{S^{2}}$ receives contributions from the residue at every point $p \in P_{I}$. This defines a cone in the space $\mathbb{R}^{s}$

$$
C(I):=\left\{\boldsymbol{r}=\sum_{i \in I} r_{i} \boldsymbol{Q}_{i} \mid r_{i}>0 \forall i \in I\right\} .
$$

The cones $C(I)$ for all $I \in \mathfrak{I}$ overlap, and a generic $\boldsymbol{r}$ lies in the interior of multiple cones. Let $\mathcal{C}$ denote the (non-empty) intersection of all cones $C(I)$ that contain $r$. The partition function, for $\boldsymbol{r} \in \mathcal{C}$, can be evaluated as

$$
Z_{S^{2}}(\boldsymbol{r})=\sum_{\substack{I \in \mathcal{I}, \boldsymbol{r} \in C(I)}} \sum_{p \in P_{I}} \operatorname{Res}_{\boldsymbol{\tau}=\boldsymbol{\tau}_{p}}\left(\sum_{\boldsymbol{m} \in \mathbb{Z}^{s}} e^{-4 \pi \boldsymbol{r} \cdot \boldsymbol{\tau}-i \boldsymbol{\theta} \cdot \boldsymbol{m}} \prod_{i=1}^{n} \frac{\Gamma\left(\frac{\mathrm{q}_{i}}{2}-\boldsymbol{Q}_{i} \cdot\left(\boldsymbol{\tau}+\frac{\boldsymbol{m}}{2}\right)\right)}{\Gamma\left(1-\frac{\mathrm{q}_{i}}{2}+\boldsymbol{Q}_{i} \cdot\left(\boldsymbol{\tau}-\frac{\boldsymbol{m}}{2}\right)\right)}\right)
$$

where $\tau_{p}$ denotes the coordinates of the point $p \in P_{I}$. The above expression is an infinite series, whose convergence is controlled by the exponential factor $e^{-4 \pi \boldsymbol{r} \cdot \boldsymbol{\tau}-i \boldsymbol{\theta} \cdot \boldsymbol{m}}$, up to a finite shift of $(\boldsymbol{r}, \boldsymbol{\theta})$ due to the exponential asymptotics of the remainder of the integrand. When $\boldsymbol{r}$ is sufficiently deep inside the cone $\mathcal{C}$ all summations are convergent. At the cone boundary, convergence is not absolute but depends on the value of $\boldsymbol{\theta}$; for particular values of $\boldsymbol{\theta}$ this series is divergent.

We thus see that the expression (2.9) for $Z_{S^{2}}$ is an integral of Mellin-Barnes type, which allows comparison of the behavior in different regions of the FI space. (See [37] for an early application of this idea to GLSMs.)

\footnotetext{
${ }^{4}$ The condition $\sum_{i=1}^{n} \boldsymbol{Q}_{i}=0$ ensures that the integrand behaves as an exponential at $\boldsymbol{\tau}=\infty$ in any wedge in $\mathbb{C}^{s}$.
} 


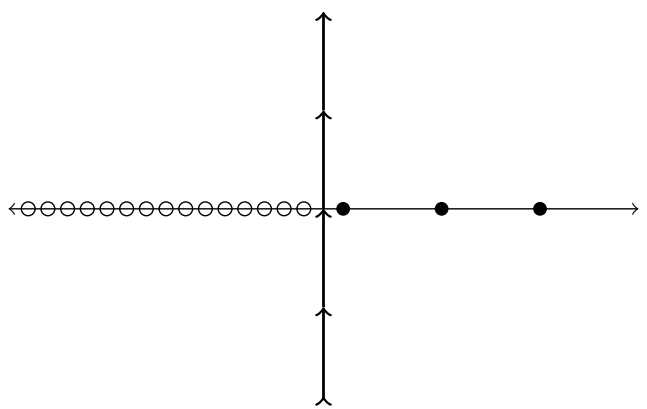

Figure 1: Pole structure of the partition function integrand for the quintic Calabi-Yau threefold GLSM. The solid black circles are fourth order poles while the unfilled circles are simple poles. The integration path is indicated and is along the imaginary axis.

\subsection{Abelian GLSM Phases and the Secondary Fan}

We next study how the behavior of the two-sphere partition function changes as we tune the value of the FI parameter $\boldsymbol{r}$.

Consider the set $\boldsymbol{C}_{\max }$ of non-empty intersections, $\bigcap_{I \in S} C(I)$ for all subsets $S \subset \mathfrak{I}$. The elements of $\boldsymbol{C}_{\max }$ are a set of non-overlapping open cones that partition the space $\mathbb{R}^{s}$. The complement of $\boldsymbol{C}_{\max }$, denoted by $\boldsymbol{B}$ is a closed subset of $\mathbb{R}^{s}$ and is a union of codimension-one hyperplanes, which we refer to as $D$-boundaries, containing the origin. A generic $\boldsymbol{r}$ lies in the interior of exactly one element, say $\mathcal{C}_{1} \in \boldsymbol{C}_{\max }$. As $\boldsymbol{r}$ is tuned it can cross a D-boundary and enter another cone $\mathcal{C}_{2} \in \boldsymbol{C}_{\max }$.

As a simple illustration, it is useful to consider the case of a GLSM for a quintic hypersurface in $\mathbb{P}^{4}$. The GLSM has $G=U(1)$ and six fields $\Phi_{i=1, \cdots, 6}$ with charges $\{1,1,1,1,1,-5\}$. The partition function integrand has poles as shown in Figure 1. The maximal cones are $\mathcal{C}_{1}=\{r>0\}$, $\mathcal{C}_{2}=\{r<0\}$, and the D-boundary is the point $r=0$. When $r>0$ we close the contour in the right half plane, where $Z_{S^{2}}$ receives contributions from the fourth-order poles, and when $r<0$, $Z_{S^{2}}$ receives contributions from the simple poles in the left half plane.

The set of cones $\mathcal{C} \in \boldsymbol{C}_{\max }$ are familiar in the context of toric geometry: they are the maximal cones in the secondary fan. Note that there are multiple descriptions of the secondary fan, as mentioned in [38, for example. Here we have recovered the Gale Transform description of the secondary fan, which utilizes GLSM charge data directly. Specifically, it is a complete fan in the space of FI parameters, whose one-dimensional cones are defined by the GLSM charges $\boldsymbol{Q}_{i=1, \cdots, n}$. The vectors $\boldsymbol{Q}_{i}$ also generate the maximal cones $\mathcal{C}$ defined above and it is a straightforward, though sometimes tedious, calculation to determine $\mathcal{C}$ explicitly. The maximal cones of the secondary fan determine the D-term phase structure of abelian GLSMs [35]. This structure is encoded in the partition function in terms of the residue sets $P_{I}$ that are contained within the domain of integration specified by $\boldsymbol{r}$.

In crossing from maximal cone $\mathcal{C}_{1}$ to $\mathcal{C}_{2}, \boldsymbol{r}$ must enter or exit some $C(I)$, and therefore, a set of residues associated to the corresponding $P_{I}$ will either enter or exit the domain of integration respectively. The residue contribution from a set $P_{I}$ can be zero if sufficiently many zero divisors $\mathfrak{H}_{i}^{(k)}$ pass through every point in $P_{I}$. When this is the case the infinite series defining the partition function does not diverge at the boundary $B$ between $\mathcal{C}_{1}$ and $\mathcal{C}_{2}$. Effectively, this erases the boundary $B$ and enlarges the maximal cone $\mathcal{C}_{1}$ to $\mathcal{C}_{1} \cup \mathcal{C}_{2}$. We will refer to D-boundaries associated 
with sets $P_{I}$ - whose residues are non zero - as Z-boundaries. This phenomenon is familiar in the study of GLSM phases. The vacuum moduli space of a GLSM can behave smoothly across a D-boundary defined by the D-terms, since it is a solution of both the D-term and the F-term equations. An example of this phenomenon occurs in certain Calabi-Yau hypersurfaces in toric varieties where a curve that is not contained in the hypersurface can be flopped. The ambient space goes through a flop while the hypersurface is unaffected. We will encounter and exploit this phenomenon is section 5.1 .

The true phase boundaries of the GLSM can be obtained by an analysis of both the D-terms and F-terms. In a number of examples, we have found that these true phase boundaries agree with the Z-boundaries defined by the partition function. However, it is conceivable that there could be phase boundaries of a GLSM which are unseen by the two-sphere partition function, although other observables suffer discontinuities. We leave the investigation of this possibility, and the relationship of the true phase boundaries and the Z-boundaries, to future work.

\subsection{Non-Abelian GLSM Phases}

To evaluate the partition function for non-abelian GLSMs we can compute the partition function of the (abelian) associated Cartan theory using the approach described above. The extra FI parameters $\tilde{r}_{\mu}, \mu=s+1, \cdots, \operatorname{rank}(\mathfrak{g})$, in the Cartan theory are useful as regulators in evaluating the necessary contour integrals.

The partition function for the Cartan theory is given by

$$
\begin{array}{r}
Z_{S^{2}}^{C}\left(r_{\mu}, \theta_{\mu}\right)=\frac{1}{|\mathcal{W}|} \sum_{m \in \mathbb{Z}^{\mathrm{rank}(\mathfrak{g})}} \int\left(\prod_{\mu=1}^{\operatorname{rank}(\mathfrak{g})} \frac{d \tau_{\mu}}{2 \pi i} e^{-4 \pi r_{\mu} \tau_{\mu}-i \theta_{\mu} m_{\mu}}\right) \\
\prod_{\alpha \in \mathcal{P}}\left(\frac{\left(\alpha_{\mu} m_{\mu}\right)^{2}}{4}-\left(\alpha_{\mu} \tau_{\mu}\right)^{2}\right) \prod_{\rho^{i} \in R_{i}} \frac{\Gamma\left(\frac{\mathbf{q}_{i}}{2}-\rho_{\mu}^{i}\left(\tau_{\mu}+\frac{m_{\mu}}{2}\right)\right)}{\Gamma\left(1-\frac{\mathrm{q}_{i}}{2}+\rho_{\mu}^{i}\left(\tau_{\mu}-\frac{m_{\mu}}{2}\right)\right)},
\end{array}
$$

where we have dropped the ${ }^{\sim}$ on the extra FI parameters $\tilde{r}_{\mu}, \mu=s+1, \cdots, \operatorname{rank}(\mathfrak{g})$. The Weyl group $\mathcal{W}$ is generated by reflections $g_{\beta}$ about the planes perpendicular to the simple roots $\beta$ of the Lie algebra. Each $g_{\beta}$ has an action on the space $\mathbb{R}^{\operatorname{rank}(\mathfrak{g})}$ and is represented by an orthogonal matrix $M_{\beta}$ [39]. Since the group elements $g_{\beta}$ permute the weights, and take all the positive roots to themselves, except for the root $\beta$ (which is mapped to $-\beta$ ), the following identity holds

$$
Z_{S^{2}}^{C}\left(r_{\mu}\left(M_{\beta}\right)_{\mu \nu}, \theta_{\mu}\left(M_{\beta}\right)_{\mu \nu}\right)=Z_{S^{2}}^{C}\left(r_{\mu}, \theta_{\mu}\right)
$$

Since the elements $g_{\beta}$, where $\beta$ is a simple root, generate $\mathcal{W}$, the above identity holds for all orthogonal matrices $M_{g}$, for $g \in \mathcal{W}$. Therefore, the phase diagram of the Cartan theory (inferred from the partition function) is symmetric under the action of the Weyl group.

The FI parameter space $\mathbb{R}^{s}$ of the non-abelian theory is the Weyl-invariant subspace of the FI parameter space $\mathbb{R}^{\operatorname{rank}(\mathfrak{g})}$ of the Cartan theory. Any phase boundary in the non-abelian theory across which the partition function $Z_{S^{2}}^{N A}$ is divergent (for some values of $\boldsymbol{\theta}$ ) must also correspond to a phase boundary in the Cartan theory. The phase boundaries of the non-abelian theory are obtained by restricting the phase diagram of the Cartan theory - and therefore its secondary fan to its Weyl-invariant subspace. We now apply this method to two examples of non-abelian GLSMs that have been studied in the literature. 
Pfaffian GLSM: This GLSM was studied in [1] and has gauge group $U(2)$. The matter content of the non-abelian theory and the Cartan theory are described below:

Associated Cartan theory

\begin{tabular}{ccc}
\multicolumn{3}{c}{ Non-abelian theory } \\
& $U(2)$ & $U(1)_{v}$ \\
\hline$\Phi_{i=1, \cdots, 7}$ & $\square$ & $2 \mathbf{q}_{\phi}$ \\
$P_{a=1, \cdots, 7}$ & $\operatorname{det}^{-1}$ & $2-2 \mathbf{q}_{\phi}$ \\
\hline
\end{tabular}

\begin{tabular}{cccc} 
& $U(1)_{1}$ & $U(1)_{2}$ & $U(1)_{v}$ \\
\hline$\Phi_{i=1, \cdots, 7}^{1}$ & 1 & 0 & $2 \mathrm{q}_{x}$ \\
$\Phi_{i=1, \cdots, 7}^{2}$ & 0 & 1 & $2 \mathrm{q}_{x}$ \\
$\Phi_{a=1, \cdots, 7}$ & -1 & -1 & $2-2 \mathrm{q}_{x}$ \\
$A_{ \pm}$ & \pm 1 & $\mp 1$ & 2 \\
\hline
\end{tabular}

The phase diagram of the non-abelian GLSM is obtained by restricting the phase diagram of the Cartan theory to the subspace $r_{1}=r_{2}$ as shown in Figure 2. Note that the $r<0$ phase of the non-abelian theory is actually a phase boundary in the associated Cartan theory. This is consistent with the fact that the restriction to $r_{1}=r_{2}$ puts the infinite sum over residues at its radius of convergence as noted in [4].

Gulliksen-Negård GLSM: The Gulliksen-Negård (GN) GLSM with gauge group $U(2) \times U(1)$ was studied in [4]. The matter content of the non-abelian and associated Cartan theories are listed below:

Associated Cartan theory

\begin{tabular}{cccc}
\multicolumn{4}{c}{ Non-abelian theory } \\
& $U(1)_{0}$ & $U(2)$ & $U(1)_{v}$ \\
\hline$\Phi_{a=1, \cdots, 8}$ & 1 & 1 & $2 \mathbf{q}_{\phi}$ \\
$X_{i=1, \cdots, 4}$ & 0 & $\square$ & $2 \mathbf{q}_{x}$ \\
$P_{i=1, \cdots, 4}$ & -1 & $\square$ & $2-2 \mathbf{q}_{x}-2 \mathbf{q}_{\phi}$ \\
\hline
\end{tabular}

\begin{tabular}{ccccc} 
Field & $U(1)_{0}$ & $U(1)_{1}$ & $U(1)_{2}$ & $U(1)_{v}$ \\
\hline$\Phi$ & 1 & 0 & 0 & $2 \mathbf{q}_{\phi}$ \\
$X_{1}$ & 0 & -1 & 0 & $2 \mathbf{q}_{x}$ \\
$X_{2}$ & 0 & 0 & -1 & $2 \mathbf{q}_{x}$ \\
$P_{1}$ & -1 & 1 & 0 & $2-2 \mathbf{q}_{x}-2 \mathbf{q}_{\phi}$ \\
$P_{2}$ & -1 & 0 & 1 & $2-2 \mathbf{q}_{x}-2 \mathbf{q}_{\phi}$ \\
$A_{ \pm}$ & 0 & \pm 1 & $\mp 1$ & 2 \\
\hline
\end{tabular}

Phase boundaries associated with changes in $Z_{S^{2}}^{N A}$ can be determined by comparison to the secondary fan of $Z_{S^{2}}^{C}$. We carry out the secondary fan analysis using only the charge vectors $\boldsymbol{Q}_{\Phi}$, $\boldsymbol{Q}_{X_{1}}, \boldsymbol{Q}_{X_{2}}, \boldsymbol{Q}_{P_{1}}, \boldsymbol{Q}_{P_{2}}$, since the one-loop determinants associated to $A_{ \pm}$do not contribute any poles. Maximal cones in the secondary fan are three-dimensional, and entering a different maximal cone by varying FI parameters requires going through a face of some cone $C(I)$. This face is itself a cone $C\left(I^{\prime}\right)$, where $I^{\prime}=\left\{\boldsymbol{Q}_{i}, \boldsymbol{Q}_{j}\right\}$ for two of the $\boldsymbol{Q}$-vectors above. Intersecting these cones with the Weyl-invariant subspace $r_{1}=r_{2}$ yields four lines and one point in $\left(r_{0}, r_{1}\right)$-space depicted in
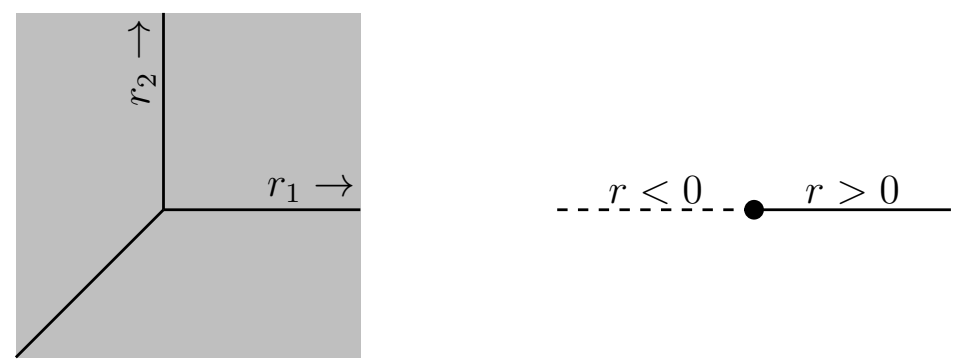

Figure 2: Phase diagram of the Pfaffian GLSM obtained by projection from the Cartan theory. 


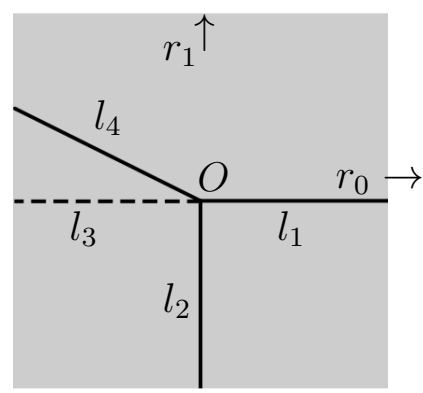

Figure 3: The candidate phase boundaries in the Gulliksen-Negård GLSM obtained by projection from the associated Cartan theory. The boundary $l_{3}$ is dashed to indicate that it is actually lifted in the IR SCFT.

Figure 3. They are given by

\begin{tabular}{c|c|c} 
locus $C\left(I^{\prime}\right) \cap\left\{r_{1}=r_{2}\right\}$ & Different Sets $I^{\prime}$ & Label \\
\hline$r_{0} \geq 0, r_{1}=0$ & $\left\{\boldsymbol{Q}_{X_{2}}, \boldsymbol{Q}_{\Phi}\right\},\left\{\boldsymbol{Q}_{X_{1}}, \boldsymbol{Q}_{\Phi}\right\}$ & line $l_{1}$ \\
$r_{0}=0, r_{1} \leq 0$ & $\left\{\boldsymbol{Q}_{X_{2}}, \boldsymbol{Q}_{X_{1}}\right\}$ & line $l_{2}$ \\
$r_{0} \leq 0, r_{1}=0$ & $\left\{\boldsymbol{Q}_{X_{1}}, \boldsymbol{Q}_{P_{1}}\right\}\left\{\boldsymbol{Q}_{X_{2}}, \boldsymbol{Q}_{P_{2}}\right\}$ & line $l_{3}$ \\
$r_{0}+2 r_{1}=0, r_{0} \leq 0$ & $\left\{\boldsymbol{Q}_{P_{2}}, \boldsymbol{Q}_{P_{1}}\right\}$ & line $l_{4}$ \\
$r_{0}=r_{1}=0$ & $\left\{\boldsymbol{Q}_{X_{1}}, \boldsymbol{Q}_{P_{2}}\right\},\left\{\boldsymbol{Q}_{X_{2}}, \boldsymbol{Q}_{P_{1}}\right\},\left\{\boldsymbol{Q}_{P_{1}}, \boldsymbol{Q}_{\Phi}\right\},\left\{\boldsymbol{Q}_{P_{2}}, \boldsymbol{Q}_{\Phi}\right\}$ & pt $O$
\end{tabular}

The phase structure of this GLSM was analyzed in [40] through a D-term and F-term analysis and revealed a phase diagram with only three boundaries $-l_{1}, l_{2}, l_{4}$. This is not an inconsistency (see discussion in 3.2) as there could be accidental cancellations of residues resulting in certain boundaries being deleted from the phase structure. In related GLSMs with gauge group $U(k) \times U(1)$, studied in [40, the boundary $l_{3}$ is a true phase boundary; determining whether this is the case requires a more detailed analysis. Nevertheless, we have seen that the secondary fan of the Cartan theory provides valuable information about phase boundaries in the Gulliksen-Negård GLSM.

\section{Geometric Phases of GLSMs}

Having discussed phase transitions, in this section we address the physics of particular phases. We describe a set of conditions on $Z_{S^{2}}$ which are necessary for a given GLSM phase to be geometric, i.e., the physics in the IR is equivalent to that of a smooth Calabi-Yau NLSM. Typically, a careful D-term and F-term analysis is necessary to ascertain whether or not a particular GLSM phase is geometric; such a phase analysis can be quite involved as illustrated by the analysis in [11]. The conditions we introduce give powerful checks on such analyses. 


\subsection{Criteria for Identifying Geometric Phases}

Let us discuss criteria for geometric phases. Consider a GLSM that flows to an $(2,2)$ SCFT with central charge $\hat{c}=3$. It satisfies [1,32]

$$
\begin{array}{ll}
\text { Condition one : } & \sum_{i=1}^{n} \operatorname{tr}_{R_{i}}(\mathfrak{t})=0, \quad \mathfrak{t} \in \mathfrak{g}, \\
\text { Condition two : } & \sum_{i=1}^{n}\left(1-\mathrm{q}_{i}\right) \operatorname{dim}\left(R_{i}\right)-\operatorname{dim}(\mathfrak{g})=\hat{c}=3 .
\end{array}
$$

The structure of the chiral ring of SCFTs associated with smooth Calabi-Yau threefolds requires [41] that gauge invariant operators have integral left and right moving R-charges. This gives

Condition three: the vector R-charge of every gauge-invariant chiral local operator $\in 2 \mathbb{Z}$,

since the chiral fields have zero axial R-charge.

Consider a phase of this GLSM defined by a region $\sum_{b=1}^{s} p_{a b} r_{b}>0$ for all $a$. This phase corresponds to a singular point in the complexified FI parameter space located at $\xi_{a}=0, a=$ $1, \cdots, s$, where $\xi_{a}:=\prod_{b=1}^{s} z_{b}^{p_{a b}}$ are local coordinates in the punctured neighborhood of the singular point. If we assume that the GLSM has a geometric phase which corresponds to a smooth CalabiYau threefold $Y$ (the point at $\xi_{a}=0$ is then called a large volume point), then the classical metric on the Kähler moduli space is specified through the following Kähler potential [19]

$$
\left.e^{-K}\right|_{\text {classical }}=\frac{1}{6} \int_{Y} J \wedge J \wedge J=\frac{1}{6} \kappa_{a b c} J_{a} J_{b} J_{c} .
$$

Here $J=\sum_{a=1}^{s} J_{a} \omega_{a}$ is the Kähler form on $Y$ that we have expanded in terms of the integral basis $\omega_{a}$ of $H^{1,1}(Y, \mathbb{Z})$, which defines the intersection form $\kappa_{a b c}:=\int_{Y} \omega_{a} \wedge \omega_{b} \wedge \omega_{c}$. Therefore, the two-sphere partition function computed in this phase must have the following asymptotic behavior in the $\xi_{a} \rightarrow 0$ limit

$$
\lim _{\xi \rightarrow 0} Z_{S^{2}}(\xi, \bar{\xi})=\frac{1}{6} \sum_{a, b, c=1}^{s} \kappa_{a b c} \log \left(\xi_{a} \bar{\xi}_{a}\right) \log \left(\xi_{b} \bar{\xi}_{b}\right) \log \left(\xi_{c} \bar{\xi}_{c}\right)
$$

since the Kähler class and FI parameters are related through $J_{a} \propto \log \left(\xi_{a} \bar{\xi}_{a}\right)$ close to a large volume point [1. Note that we have absorbed any positive numerical constants in (4.5) using a Kähler transformation. This leads us to

Condition four: $Z_{S^{2}}$ must have the asymptotics as in equation (4.5)

which is a necessary condition for the existence of a geometric phase.

Let us briefly discuss some relevant background before stating our final criterion necessary for the existence of a geometric phase. Any $\hat{c}=3$ SCFT satisfying condition (4.3) can be used as the worldsheet theory of the type II superstring to generate a four-dimensional $\mathcal{N}=2$ supergravity [42,43] . The $s$-dimensional complexified FI parameter space $\mathcal{M}$ is governed by special geometry [16 22]. Let $\left(\xi_{a}\right), a=1, \cdots, s$ be local coordinates on $\mathcal{M}$. Special geometry implies that $\mathcal{M}$ is a Hodge-Kähler manifold that carries an $S p(2 s+2, \mathbb{Z})$ bundle with local sections $X^{I}(\xi), I=$ $0, \cdots, s$. In addition, there exists a holomorphic function $\mathcal{F}(X)$, known as the prepotential that is 


\begin{tabular}{|l|l|}
\hline 1. & $\sum_{i=1}^{n} \operatorname{tr}_{R_{i}}(\mathfrak{t})=0, \quad \mathfrak{t} \in \mathfrak{g}$. \\
2. & $\sum_{i=1}^{n}\left(1-\mathbf{q}_{i}\right) \operatorname{dim}\left(R_{i}\right)-\operatorname{dim}(\mathfrak{g})=\hat{c}=3$. \\
3. & $\begin{array}{l}\text { The vector R-charge of every gauge-invariant chiral local operator is } \\
\text { an even integer. }\end{array}$ \\
4. & $\lim _{\xi \rightarrow 0} Z_{S^{2}}(\xi, \bar{\xi})=\frac{1}{6} \sum_{a, b, c=1}^{s} \kappa_{a b c} \log \left(\xi_{a} \bar{\xi}_{a}\right) \log \left(\xi_{b} \bar{\xi}_{b}\right) \log \left(\xi_{c} \bar{\xi}_{c}\right)$. \\
\hline
\end{tabular}

Table 1: The geometric phase criteria.

homogeneous and has degree 2. Locally, $\left(X^{I}, \frac{\partial \mathcal{F}}{\partial X^{I}}\right)$ specify a choice of frame for the $S p(2 s+2, \mathbb{Z})$ bundle and are known as the periods. The Kähler potential on moduli space is specified through the periods as

$$
e^{-K(\xi, \bar{\xi})}=i\left(X^{I} \frac{\overline{\partial \mathcal{F}}}{\partial X^{I}}-\overline{X^{I}} \frac{\partial \mathcal{F}}{\partial X^{I}}\right)
$$

For definitions and further details about special geometry we refer the reader to [20,22].

When the GLSM is in a geometric phase the periods $\left(X^{I}, \partial_{I} \mathcal{F}\right)$ have a special structure: in the $\xi_{a} \rightarrow 0$ limit, the leading behavior is given by

$$
\begin{gathered}
X^{0} \sim \prod_{a=1}^{s} \xi_{a}^{\delta_{a}}, \quad X^{b} \sim X^{0}(\xi) \log \xi_{b}, \quad \partial_{b} F \sim X^{0}(\xi) C_{a b c} \log \xi_{b} \log \xi_{c} \\
\partial_{0} F \sim X^{0}(\xi) C_{a b c} \log \xi_{a} \log \xi_{b} \log \xi_{c}, \quad b=1, \cdots, s
\end{gathered}
$$

where $\delta_{a} \in \mathbb{Q}$. This behavior of the periods as $\xi \rightarrow 0$ is usually referred to as maximally unipotent monodromy (MUM), which is a statement about the form of the $S p(2 s+2, \mathbb{Z})$ monodromy around any boundary divisor passing through $\xi=0$ [44,45].

Recall the evaluation of the partition function by the method of residues discussed in Section 3.1 . Consider all $r k(\mathfrak{g})$-element subsets $I \subset\{1, \cdots, n\}$ such that the corresponding pole hyperplanes intersect in a point set $P_{I}$. Let $\mathcal{I}_{0}$ denote the set of all subsets $I$ whose point sets $P_{I}$ lie inside the contours of integration defined by the GLSM phase. The partition function is evaluated by adding up all the residues at the points in $\bigcup_{I \in \mathcal{I}_{0}} P_{I}$. If $P_{I} \neq P_{J}$ for some $I, J \in \mathcal{I}_{0}$ the contributions to the partition function from the two sets of residues will involve distinct power series expansions. We suspect that this signals the presence of at least two independent periods, which in the $\xi \rightarrow 0$ limit do not diverge as a power of $\log \xi_{a}$. If this is the case, the periods do not have the log-structure as in equation (4.8) that is characteristic of MUM. We are led to a condition

$$
P_{I}=P_{J} \quad \forall I, J \in \mathcal{I}_{0}
$$

We would like to stress that we do not have a proof that this condition on the residue structure of the partition function is necessary for MUM. We will return to this condition in the context of a particular example in Section 4.3 .

We have discussed four necessary conditions for the existence of a geometric phase in a GLSM. We refer to these as the geometric phase criteria and for convenience list them in Table 1 . 


\section{Geometric Phase Criteria for abelian GLSMs}

For an abelian GLSM one of these criteria is equivalent to a constraint on the partition function integrand which is easy to check. Recall that the partition function for an abelian GLSM is given in equation (2.9). Each one-loop matter determinant $Z_{\phi_{i}}$ has a pole along the hyperplanes $H_{i}^{(k)}$ (polar divisors) and a zero along the hyperplanes $\mathfrak{H}_{i}^{(k)}$ (zero divisors). Consider a point $p \in P_{I}$, for some $I \in \mathcal{I}_{0}$, located at the codimension- $s$ intersection of the polar divisors $H_{i}^{\left(k_{i}\right)}$ for some $k_{i}$ and all $i \in I$. Let $S_{p}$ and $S_{z}$ be the total number of polar divisors and zero divisors containing $p$, respectively. Then we define the multiplicity $\mathrm{m}$ of $p$ to be $\mathrm{m}=S_{p}-S_{z}-s+1$. When $\mathrm{m}>1$ we expand the integrand in a multidimensional Laurent series and read off the residue as the coefficient of the $\prod_{a=1}^{s} \frac{1}{\tau_{a}-\tau_{a}^{(p)}}$ term, where $\boldsymbol{\tau}^{(p)}$ are the coordinates of $p$. In doing so we have to take $\mathrm{m}-1$ derivatives of the integrand in (2.9). Each derivative $\partial / \partial \tau_{a}$ results in a $\log z_{a}$ term in the residue. As a simple illustration of the above phenomenon, when $s=1$ the multiplicity at $p$ is simply the order of the pole at $p$. For a pole of order $\mathrm{m}$,

$$
\operatorname{Res}_{\tau=0} \frac{f(\tau)}{\tau^{\mathrm{m}+1}}=\left.\frac{1}{\mathrm{~m} !} \frac{d^{\mathrm{m}} f}{d \tau^{\mathrm{m}}}\right|_{\tau=0}
$$

If $f(\tau)$ contained a factor of $z^{\tau}$ the derivatives above would generate a $(\log z)^{\mathrm{m}-1}$ term.

Given this relationship between $\mathrm{m}$ and the log structure of the partition function, it is easy to see that for an abelian GLSM criterion 4 in Table 1 is equivalent to the condition:

there exists a point $p \in P_{I}$ for some $I$ that has multiplicity $\mathrm{m}=4$,

which is very easy to check in examples.

\subsection{Are the criteria sufficient?}

We present a simple example that shows that the criteria in Table 1 are necessary but not sufficient conditions for the existence of a geometric phase. Consider a GLSM with gauge group $U(1) \times \mathbb{Z}_{5}$ and matter content as shown:

\begin{tabular}{|c|ccccc|}
\hline Field & $X_{1,2,3,4}$ & $P_{1}$ & $P_{2}$ & $P_{3}$ & $P_{4}$ \\
\hline$U(1)$ & +1 & -1 & -1 & -1 & -1 \\
$U(1)_{R}$ & 0 & $\frac{2}{5}$ & $\frac{4}{5}$ & $\frac{6}{5}$ & $\frac{8}{5}$ \\
\hline
\end{tabular}

The $\mathbb{Z}_{5}$ gauge symmetry acts as $P_{j} \rightarrow e^{2 \pi i j / 5} P_{j}, j=1, \cdots, 4$. The most general superpotential is

$$
W=P_{2} P_{3} G_{1}(X)+P_{1} P_{4} G_{2}(X)+P_{1} P_{2}^{2} G_{3}(X)+P_{1}^{2} P_{3} G_{4}(X)+P_{1}^{3} P_{2} G_{5}(X)+P_{1}^{5} G_{6}(X),
$$

where $G_{i}(X)$ are homogeneous polynomials whose degrees are fixed by gauge invariance.

The two-sphere partition function in the $r>0$ phase is

$$
Z_{S^{2}}=\sum_{n, l=0}^{\infty} \oint \frac{d \epsilon}{2 \pi i} \frac{\pi^{4}}{\sin ^{4} \pi \epsilon} z^{n-\epsilon} \bar{z}^{l-\epsilon} \prod_{k=1}^{4} \frac{\Gamma\left(\frac{k}{5}+n-\epsilon\right) \Gamma\left(\frac{k}{5}+l-\epsilon\right)}{\Gamma(1+n-\epsilon) \Gamma(1+l-\epsilon)} .
$$




\begin{tabular}{|c|cccccc|}
\hline field & $\phi_{1}$ & $\phi_{2}$ & $\phi_{3}$ & $\phi_{4}$ & $\phi_{5}$ & $P$ \\
\hline$U(1)$ & 1 & $w_{2}$ & $w_{3}$ & $w_{4}$ & $w_{5}$ & $-1-\sum_{i=2}^{5} w_{i}$ \\
$U(1)_{R}$ & 0 & 0 & 0 & 0 & 0 & 2 \\
\hline
\end{tabular}

Table 2: This table lists the field content of GLSMs that model a degree $\sum_{i=1}^{5} w_{i}$ hypersurface in the weighted projective space $\mathbb{P}^{w_{1}, \cdots, w_{5}}$.

In fact, via $\Gamma$-function identities the expression above is equal to the partition function of a GLSM for a quintic hypersurface in $\mathbb{P}^{4}$ that was discussed briefly in Section 3.2. The partition function only has poles of fourth order and all the geometric phase criteria are met in this GLSM.

The vacuum moduli space of the GLSM is a solution to the D-term and F-term equations:

$$
\begin{gathered}
\left|x_{1}\right|^{2}+\left|x_{2}\right|^{2}-\left|p_{1}\right|^{2}-\left|p_{2}\right|^{2}=r \\
p_{1} G_{2}(x)=0, \quad p_{2} G_{1}(x)+p_{1}^{2} G_{4}(x)=0, \quad p_{3} G_{1}(x)+2 p_{1} p_{2} G_{3}(x)+p_{1}^{3} G_{5}(x)=0 \\
p_{4} G_{2}(x)+p_{2}^{2} G_{3}(x)+2 p_{1} p_{3} G_{4}(x)+3 p_{1}^{2} p_{2} G_{5}(x)+5 p_{1}^{4} G_{6}(x)=0 \\
p_{2} p_{3} \partial_{i} G_{1}(x)+p_{1} p_{4} \partial_{i} G_{2}(x)+p_{1} p_{2}^{2} \partial_{i} G_{3}(x)+p_{1}^{2} p_{3} \partial_{i} G_{4}(x)+p_{1}^{3} p_{2} \partial_{i} G_{5}(x)+p_{1}^{5} \partial_{i} G_{6}(x)=0
\end{gathered}
$$

When $r>0$ we must have $x \neq 0$. It is easy to see that the classical vacuum moduli space has a non-compact branch of solutions:

$$
p_{1}=0, \quad p_{2}=0, \quad G_{1}(x)=0, \quad G_{2}(x)=0, \quad p_{3}, p_{4} \text { arbitrary. }
$$

Consequently, the low-energy field theory is singular and cannot be identified with a compact Calabi-Yau NLSM, even though all the geometric phase criteria were satisfied. It is intriguing that in spite of this singular behavior, the procedure outlined in [4] can be used to extract the GromovWitten invariants, which agree with those of the quintic Calabi-Yau threefold. This example illustrates that the criteria are not sufficient to ensure a geometric interpretation of the low-energy physics as a NLSM with a compact Calabi-Yau threefold as target space.

\subsection{The Geometric Criteria and Singular Geometries}

In this section we study the geometric phase criteria in GLSM phases, which correspond to singular limits of Calabi-Yau NLSMs. These serve as examples of GLSM phases that satisfy the conditions in Table 1, but fail criterion (4.9).

Consider the class of GLSMs defined by the field content in Table 2 and the superpotential $W=P G(\phi)$, where $G$ is a quasi-homogeneous polynomial of degree $\sum_{i=1}^{5} w_{i}$. The models with $\left(w_{1}, w_{2}, w_{3}, w_{4}, w_{5}\right)$ given by $(1,1,1,1,2),(1,1,1,1,4)$ and $(1,1,1,2,5)$ have singularities at points in the ambient weighted project space that miss the generic hypersurface $G(\phi)=0$. Therefore, these models have a geometric phase that can be interpreted as a NLSM with a smooth Calabi-Yau threefold as target space. All the criteria in Table 1 are satisfied in these examples.

The ambient space singularities for the the models given in Table 3 have singularities on curves and/or surfaces that intersect the generic hypersurface. These singularities can be resolved through blowups of the ambient space and result in smooth Calabi-Yau threefolds with two or three moduli [29, 46, 47]. The $r>0$ phase of these GLSMs satisfies the first four criteria listed in Table 1, in particular, the partition function has the right asymptotics as $z \rightarrow 0$. In each case, however, there 


\begin{tabular}{|c|c|c|c|}
\hline$\left(w_{1}, w_{2}, w_{3}, w_{4}, w_{5} \mid-\sum_{i=1}^{5} w_{i}\right)$ & $h^{1,1}$ & Distinct $P_{I}$ & $2 h^{1,1}+2=\sum_{p \in\{\text { box }\}} \mathrm{m}_{p}$ \\
\hline$(1,1,2,2,2 \mid-8)$ & 2 & 2 & $4+2$ \\
$(1,1,2,2,6 \mid-12)$ & 2 & 2 & $4+2$ \\
$(1,2,2,3,4 \mid-12)$ & 2 & 2 & $4+2$ \\
$(1,2,2,2,7 \mid-14)$ & 2 & 2 & $4+2$ \\
$(1,1,1,6,9 \mid-18)$ & 2 & 2 & $4+1+1$ \\
\hline$(1,1,1,3,6 \mid-12)$ & 3 & 3 & $4+2+2$ \\
$(1,2,3,3,3 \mid-12)$ & 3 & 3 & $4+2+2$ \\
$(1,3,3,3,5 \mid-15)$ & 3 & 3 & $4+2+2$ \\
$(1,2,3,3,9 \mid-18)$ & 3 & 3 & $4+2+2$ \\
$(1,1,2,8,12 \mid-24)$ & 3 & 3 & $4+1+2+1$ \\
\hline
\end{tabular}

Table 3: The first column defines the GLSM through its $U(1)$-charges. The second column is the number of Kähler moduli in the resolved geometry. The third column denotes the number of distinct $P_{I}$ whose residues are non-zero and that contribute to the contour integral. The fourth column demonstrates the relationship between pole multiplicities and $2 h^{1,1}+2$.

are non-zero residue contributions to $Z_{S^{2}}$ arising from multiple, distinct sets $P_{I}$. Interestingly, the number of such distinct $P_{I}$ exactly equals the number of Kähler moduli in the resolved threefold. It is tempting to speculate that a GLSM phase that satisfies the geometric phase criteria, but not condition (4.9), may signal a singular geometry of the kind encountered above.

Moreover, a careful analysis of the pole structure using the method of appendix B reveals that, within a given modular box in the singular GLSM partition function, the sum of the multiplicities $\mathrm{m}$ for contributing poles is precisely $2 h^{1,1}+2$, where $h^{1,1}$ is the number of Kähler moduli in the resolved geometry. This matches the $2 h^{1,1}+2$ periods that enter the partition function in the resolved GLSM, and the fact that the counting matches the unresolved GLSM suggests that the partition function captures a singular limit of the periods.

\section{Illustrative Examples}

In this section we apply the geometric phase criteria in Table 1 and identify novel geometric phases of GLSMs. We study a collection of GLSMs with gauge group $U(1)$ and charged matter fields that satisfy the above criteria. We focus our attention on one member of the collection, which we call the $P^{2}$-GLSM, and perform an analysis of the D-term and the F-terms. The phase that satisfies the geometric criteria appears as a hybrid phase $Y$, which can be visualized semi-classically as two copies of $\mathbb{P}^{3}$ glued together by a Landau-Ginzburg model. We provide a physical argument that shows that this hybrid phase can be identified with an NLSM with target space $X$, the doublecover of $\mathbb{P}^{3}$ branched over a smooth octic surface. The argument involves studying the phase diagram of an auxiliary GLSM, which we call GLSM2, with gauge group $U(1) \times U(1)$. GLSM2 has three phases: the hybrid phase $Y$, a geometric phase corresponding to the Calabi-Yau threefold $X$ and a Landau-Ginzburg orbifold phase. We show that the IR physics of GLSM2 is identical along a codimension 1 locus in the FI parameter space, and this locus connects the singular points corresponding to the geometric phase $X$ and the hybrid phase $Y$. We believe this to be a strong physical argument for a geometric interpretation of the hybrid phase of the $P^{2}$-GLSM and the 
other GLSMs in the collection.

\subsection{Calabi-Yau Geometries from Gauge Theories}

We consider a special class of GLSMs with no $U(1)_{A}$ anomaly that flow to $(2,2)$ SCFTs with $\hat{c}=3$ and integral R-charges. The gauge group is $G=U(1) \times \prod_{a=1}^{n_{p}} \mathbb{Z}_{m_{a}}$, while the matter content consists of $n$ fields $\Phi_{i}$ and $n_{p}$ fields $P_{a}$, carrying $U(1)$ charges +1 and $-k_{a}$ respectively. The superpotential is

$$
W=\sum_{a=1}^{n_{p}} P_{a}^{m_{a}} G_{m_{a} k_{a}}(\Phi) .
$$

where $G_{m_{a} k_{a}}(\Phi)$ is the most general degree $m_{a} k_{a}$ polynomial in $\Phi_{i}$. The Lagrangian has a discrete symmetry

$$
P_{a} \rightarrow e^{2 \pi i / m_{a}} P_{a}, \forall a=1, \cdots, n_{p},
$$

which we declare to be a gauge symmetry. Here $m_{a}, k_{a} \geq 1$ and are integral with the condition that if $m_{a}=1$ then $k_{a} \geq 2$. Note that this class of GLSMs allows the " $P$ fields" to appear with general exponents in the superpotential, in contrast to the standard GLSM construction for complete intersections in toric varieties. The R-charge of $\Phi_{i}$ is assumed to be $\mathrm{q}_{\phi}>0$ and consequently $P_{a}$ has R-charge $\mathrm{q}_{a}=2 / m_{a}-k_{a} \mathbf{q}_{\phi}$. The anomaly and central charge conditions read

$$
n=\sum_{a=1}^{n_{p}} k_{a}, \quad 3=n-1+n_{p}-2 \sum_{a=1}^{n_{p}} \frac{1}{m_{a}} .
$$

The discrete gauge symmetry is important to ensure that gauge-invariant chiral operators carrying fractional left/right-moving R-charges, e.g., $\mathcal{O}=P_{a} \Phi_{i}^{k_{a}}$, are projected out.

We apply the criteria in Table 1 and look for geometries in the $r \gg 0$ phase, i.e., in the neighborhood of the singular point at $z=0$ (recall that $z=e^{-2 \pi r+i \theta}$ ). The $S^{2}$ partition function for this class of GLSMs is given by

$$
Z_{S^{2}}=\sum_{m \in \mathbb{Z}} \int_{\frac{\mathrm{q}_{\phi}}{2}-i \infty}^{\frac{\mathrm{q}_{\phi}}{2}-i \infty} \frac{d \tau}{2 \pi i} z^{\tau-m / 2} \bar{z}^{\tau+m / 2} \frac{\Gamma\left(-\tau-\frac{m}{2}\right)^{n}}{\Gamma\left(1+\tau-\frac{m}{2}\right)^{n}} \prod_{a=1}^{n_{p}} \frac{\Gamma\left(\frac{1}{m_{a}}+k_{a}\left(\tau+\frac{m}{2}\right)\right)}{\Gamma\left(1-\frac{1}{m_{a}}+k_{a}\left(-\tau+\frac{m}{2}\right)\right)} .
$$

Near $z=0$, the partition function can be evaluated by closing the contour in the right half plane, thereby receiving contributions from the poles at $\tau=-m / 2+n$, with $n \geq 0, m \leq n$. This series of poles has order $n-\ell$, where $\ell$ denotes the number of $P_{a}$ with exponent $m_{a}=1$. (The $\Gamma$ functions associated to these fields produce zeros that reduce the pole order from $n$.) The geometric criteria imply that

$$
n-\ell=4 .
$$

There are only finitely many GLSMs in this class that satisfy conditions (5.3) and (5.5). The possibilities are listed in Table 4 . 


\begin{tabular}{|c|c|c|c|}
\hline$n$ & $n_{p}$ & $P$-field charges $\left(k_{a}\right)$ & Exponents in $W\left(m_{a}\right)$ \\
\hline 4 & 4 & $(-1,-1,-1,-1)$ & $(2,2,2,2)$ \\
\hline 4 & 3 & $(-2,-1,-1)$ & $(2,2,2)$ \\
5 & 4 & $(-2,-1,-1,-1)$ & $(1,2,2,2)$ \\
\hline 4 & 2 & $(-3,-1)$ & $(2,2)$ \\
5 & 3 & $(-3,-1,-1)$ & $(1,2,2)$ \\
4 & 2 & $(-2,-2)$ & $(1,2)$ \\
5 & 3 & $(-2,-2,-1)$ & $(1,1,2,2)$ \\
6 & 4 & $(-2,-2,-1,-1)$ & $(2)$ \\
\hline 4 & 1 & $(-4)$ & $(2,1)$ \\
5 & 2 & $(-1,-4)$ & $(1,2)$ \\
5 & 2 & $(-2,-3)$ & $(1,1,2)$ \\
5 & 2 & $(-2,-3)$ & $(1,1,2)$ \\
6 & 3 & $(-2,-2,-2)$ & $(1,1,1,2)$ \\
6 & 3 & $(-3,-2,-1)$ & $(1)$ \\
7 & 4 & $(-2,-2,-2,-1)$ & $(1,1)$ \\
\hline \hline 5 & 1 & $(-5)$ & $(1,1)$ \\
6 & 2 & $(-3,-3)$ & $(1,1,1)$ \\
6 & 2 & $(-2,-4)$ & \\
7 & 3 & $(-2,-2,-3)$ & \\
8 & 4 & $(-2,-2,-2,-2)$ & \\
\hline
\end{tabular}

Table 4: Matter content of GLSMs satisfying the MUM criteria. The last five solutions are complete intersection Calabi-Yau varieties in $\mathbb{P}^{n-1}$.

\section{Is the $r>0$ phase geometric?}

This leads to the question of whether the singular point at $z=0$ is a geometry. The partition function for a GLSM in Table 4 is

$Z_{S^{2}}=\sum_{m \in \mathbb{Z}} \int_{\frac{-\mathrm{q}_{\phi}}{2}-i \infty}^{\frac{-\mathrm{q}_{\phi}}{2}-i \infty} \frac{d \tau}{2 \pi i} e^{-4 \pi r \tau-i \theta m} \frac{\Gamma\left(-\tau-\frac{m}{2}\right)^{n}}{\Gamma\left(1+\tau-\frac{m}{2}\right)^{n}} \prod_{\mu=1}^{\ell} \frac{\Gamma\left(1+k_{\mu}\left(\tau+\frac{m}{2}\right)\right)}{\Gamma\left(k_{\mu}\left(-\tau+\frac{m}{2}\right)\right)} \prod_{\alpha=1}^{n_{p}-\ell} \frac{\Gamma\left(\frac{1}{2}+k_{\alpha}\left(\tau+\frac{m}{2}\right)\right)}{\Gamma\left(\frac{1}{2}+k_{\alpha}\left(-\tau+\frac{m}{2}\right)\right)}$.

Note that we have split the product over $n_{p}$ fields depending on whether the corresponding $P_{a}$ have $m_{a}=1$ or $m_{a}=2$. Using $\Gamma$ function identities, the integrand can be transformed to

$$
\begin{gathered}
Z_{S^{2}}=\sum_{m \in \mathbb{Z}} \int_{\frac{-\mathrm{q}_{\phi}}{2}-i \infty}^{\frac{-\mathrm{q}_{\phi}}{2}-i \infty} \frac{d \tau}{2 \pi i} e^{-4 \pi r^{\prime} \tau-i \theta^{\prime} m} \frac{\Gamma\left(-\tau-\frac{m}{2}\right)^{n}}{\Gamma\left(1+\tau-\frac{m}{2}\right)^{n}} \prod_{\alpha=1}^{n_{p}-\ell} \frac{\Gamma\left(-k_{\alpha}\left(\tau+\frac{m}{2}\right)\right)}{\Gamma\left(1+k_{\alpha}\left(\tau-\frac{m}{2}\right)\right)} \\
\prod_{\mu=1}^{\ell} \frac{\Gamma\left(1+k_{\mu}\left(\tau+\frac{m}{2}\right)\right)}{\Gamma\left(k_{\mu}\left(-\tau+\frac{m}{2}\right)\right)} \prod_{\alpha=1}^{n_{p}-\ell} \frac{\Gamma\left(1+2 k_{\alpha}\left(\tau+\frac{m}{2}\right)\right)}{\Gamma\left(2 k_{\alpha}\left(-\tau+\frac{m}{2}\right)\right)},
\end{gathered}
$$

where

$$
r^{\prime}=r+\frac{\log 2}{\pi} \sum_{\alpha=1}^{n_{p}-\ell} k_{\alpha}, \quad \theta^{\prime}=\theta+\sum_{\alpha=1}^{n_{p}-\ell} k_{\alpha} \pi
$$


We note that (5.6) is precisely the partition function of a GLSM for a complete intersection of $n_{p}$ hypersurfaces, of degrees $k_{\mu=1}, \cdots, k_{\mu=\ell}$ and $2 k_{\alpha=1}, \cdots, 2 k_{\alpha=n_{p}-\ell}$, in the weighted projective space $Y=\mathbb{P}\left[1,1, \cdots, 1, k_{\alpha=1}, \cdots, k_{\alpha=n_{p}-\ell}\right]$ of dimension $n+n_{p}-\ell-1=n_{p}+3$. The singular locus of the ambient space $Y$ is at least codimension $n$ and, consequently, does not intersect with the $n_{p}$ defining hypersurface equations. The complete intersection defines a smooth Calabi-Yau threefold geometry.

We have found that the partition function of each GLSM in the collection, as an expansion around $z=0$, agrees with the partition function of a geometric complete intersection, as an expansion around the large volume point. The equality of the partition functions implies that the Gromov-Witten invariants in the $r>0$ phase must agree with those of the corresponding complete intersection Calabi-Yau threefold [4]. This is evidence in favor of a geometric interpretation of the $r>0$ phase of these GLSMs. We will specialize to one solution in Table 4 and argue more convincingly for a geometric interpretation of the $r>0$ phase. It is likely that this argument generalizes to all the solutions in Table 4, but we will restrict ourselves to this one example for the sake of simplicity.

\section{The $P^{2}$-GLSM describes a geometry}

The GLSM we study, which we will refer to as the " $P^{2}$-GLSM", has gauge group $U(1) \times \mathbb{Z}_{2}$ and matter consisting of superfields $X_{i}$ with charge +1 and a field $P$ of charge -4 ; and under the $\mathbb{Z}_{2}$ gauge symmetry, $P \rightarrow-P$. The superpotential takes the form

$$
W=P^{2} G_{8}(X),
$$

where $G_{8}(x)$ is a generic homogeneous polynomial of degree 8 .

We first analyze the phase structure of this GLSM. The classical vacuum moduli space is a solution of the equations

$$
\begin{gathered}
\text { D-term } \\
\sum_{i=1}^{4}\left|x_{i}\right|^{2}-4|p|^{2}=r \quad p G_{8}(x)=0, \quad p^{2} \partial_{i} G_{8}=0 .
\end{gathered}
$$

When $r<0$ we have $p \neq 0$, which breaks the gauge group from $U(1) \times \mathbb{Z}_{2}$ down to a $\mathbb{Z}_{8}$, generated by the element $\left(e^{2 \pi i / 8},-1\right) \subset U(1) \times \mathbb{Z}_{2}$. The $X_{i}$ are massless and interact through the effective superpotential $W=\langle P\rangle^{2} G_{8}(X)$. This phase is a Landau-Ginzburg orbifold.

When $r>0$, we must have $x \neq 0$. Since $G_{8}(x)$ is generic we must have $p=0$. The classical vacuum moduli space is a $\mathbb{P}^{3}$ spanned by the $x_{i}$ with an unbroken $\mathbb{Z}_{2}$ gauge symmetry. However, this classical analysis is insufficient, as was pointed out in [12 14] in a closely related example. Thinking semi-classically, although we have $\langle p\rangle=0$, the field becomes massless and fluctuates along the octic locus $\left(G_{8}(x)=0\right) \subset \mathbb{P}^{3}$. Away from this locus, $p$ can be integrated out, leaving an unbroken $\mathbb{Z}_{2}$ gauge symmetry. A $\mathbb{Z}_{2}$ gauge theory has two bosonic vacua, and thus the moduli space of vacua - away from the octic locus - consists of two copies of $\mathbb{P}^{3}$. This phase is referred to as a true hybrid phase in the literature since the $\mathbb{P}^{3}$ is fixed by the R-symmetry [48]. 


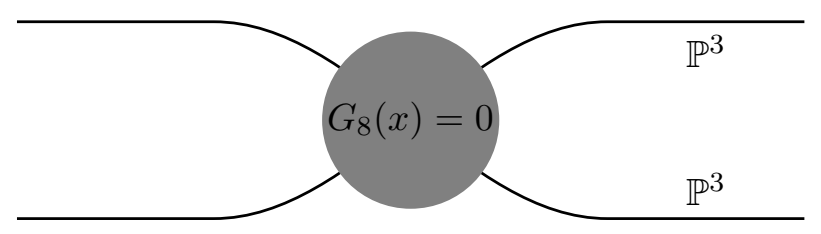

This picture suggests that this phase may be related to a Calabi-Yau threefold that is obtained by taking a double-cover of $\mathbb{P}^{3}$ branched over an octic surface. We have shown above that the $S^{2}$ partition function in this phase is equal to the partition function of a degree 8 hypersurface in $\mathbb{P}^{1,1,1,1,4}$ with homogeneous coordinates $\left[x_{1}, x_{2}, x_{3}, x_{4}, y\right]$. A special family of hypersurfaces of the form

$$
y^{2}=G_{8}(x),
$$

demonstrates that this Calabi-Yau threefold, on a sublocus of its complex structure moduli space, can indeed be viewed as a double-cover of $\mathbb{P}^{3}$ branched over an octic surface. The weighted projective space $\mathbb{P}^{1,1,1,1,4}$ has singularities at points, which are missed by the generic hypersurface. For generic $G_{8}(x)$ the Calabi-Yau threefold is smooth.

We will now show that the $r>0$ phase is geometric. To do so we consider a new GLSM, which we will refer to as GLSM2, with gauge group $U(1) \times U(1)$ and matter content given below.

\begin{tabular}{|c|cccc|}
\hline Field: & $X_{i}$ & $Y$ & $Q$ & $P$ \\
\hline$U(1)_{1}$ & +1 & 4 & 0 & -8 \\
\hline$U(1)_{2}$ & 0 & 1 & 1 & -2 \\
\hline
\end{tabular}

GLSM2 has a superpotential

$$
W=P\left(Y^{2}-Q^{2} G_{8}(X)\right) .
$$

The classical vacuum moduli space is a solution to the equations

$$
\begin{array}{cl}
\text { D-terms } & \multicolumn{1}{c}{\text { F-terms }} \\
\sum_{i=1}^{4}\left|x_{i}\right|^{2}+4|y|^{2}-8|p|^{2}=r_{1}, & y^{2}-q^{2} G_{8}(x)=0, \quad p y=0, \\
|y|^{2}+|q|^{2}-2|p|^{2}=r_{2}, & p q G_{8}(x)=0, \quad p q^{2} \partial_{i} G_{8}=0 .
\end{array}
$$

The low-energy effective theory is a function of $\left(r_{1}, r_{2}\right)$ and is depicted in Figure 4 . The phase boundaries in figure 4 are the one-dimensional rays generated by the charge vectors $\boldsymbol{Q}_{i}=(1,0)$, $\boldsymbol{Q}_{Q}=(0,1)$ and $\boldsymbol{Q}_{P}=(-8,-2)$, agreeing with the secondary fan discussion of section ${ }_{3.2}^{5}$

We describe the phases as:

- $r_{1}>0, r_{2}>0$ : The equations (5.12) imply that $q \neq 0$ and $x \neq 0$. Consequently, we have $p=0$ for generic $G_{8}(x)$. Since $q \neq 0$ we can use the $\mathbb{C}^{*}$ action associated with $U(1)_{2}$ to set $q=1$. The vacuum moduli space is given by the Calabi-Yau hypersurface $X:\left(y^{2}-G_{8}(x)=0\right)$ in $\mathbb{P}^{1,1,1,1,4}$, which is a double-cover of $\mathbb{P}^{3}$ branched over the octic $G_{8}(x)=0$.

- $r_{2}<0, r_{1}-4 r_{2}>0$ : We have $x \neq 0$ and $p \neq 0$, which implies that $y=0$ and $q=0$. Using the D-term we can set $\langle p\rangle=\sqrt{\left|r_{2}\right| / 2}$. The field $Y$ can be integrated out since it has a mass proportional to $\sqrt{\left|r_{2}\right|}$. The low-energy effective theory consists of the fields $Q$ and $X_{i}$

\footnotetext{
${ }^{5}$ The secondary fan also contains the ray $Q_{Y}=(4,1)$, which is not a real phase boundary as far as the IR physics is concerned due to the F-terms. From the partition function perspective, the associated poles in $Z_{Y}$ are cancelled by zeroes of $Z_{P}$.
} 


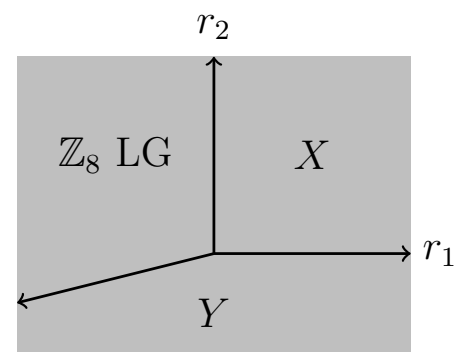

Figure 4: Phases of GLSM2.

interacting via a superpotential

$$
W_{e f f}=\sqrt{\left|r_{2}\right| / 2} Q^{2} G_{8}(X)
$$

The expectation value of $P$ breaks the gauge group from $U(1) \times U(1)$ to $U(1) \times \mathbb{Z}_{2}$, where the $Z_{2}$ acts as $Q \rightarrow-Q$. This is precisely the $r>0$ phase of the $P^{2}$-GLSM! We will denote this phase by $Y$, which can be imagined as two copies of $\mathbb{P}^{3}$ "glued together" by an interacting Landau-Ginzburg theory.

- $r_{1}<0, r_{1}-4 r_{2}<0$ : We have $q \neq 0$ and $p \neq 0$. The gauge group is broken to $\mathbb{Z}_{8}$ and we have the Landau-Ginzburg orbifold phase that we encountered earlier as the $r<0$ phase of the $P^{2}$-GLSM.

GLSM2 in the $r_{1}>0, r_{2}>0$ phase describes a hypersurface in a toric variety, which is a resolution of the singularities of the weighted projective space $\mathbb{P}_{1,1,1,1,4}$. Since the generic hypersurface misses the singular points in $\mathbb{P}_{1,1,1,1,4}$ it is insensitive to the details of the resolution and therefore the IR SCFT associated to this phase of GLSM2 must depend on only one combination of FI parameters. The two-sphere partition function must reflect this fact, and upon computing it we find that the relevant combination is

$$
w=\frac{2^{8} z_{1}}{\left(1-4 z_{2}\right)^{4}}
$$

We emphasize that the IR physics is the same along the loci $w=$ constant. Since one such locus connects the boundary points associated to phases $X$ (the geometric phase) and $Y\left(P^{2}\right.$-GLSM hybrid phase), these phases have identical IR physics and therefore the hybrid theory describes a geometry.

We believe that a similar analysis will hold for the GLSMs listed in Table 4, and therefore they should describe Calabi-Yau threefold geometries in the $r>0$ phase.

\section{GKZ systems for GLSMs}

In this section we prove that the two-sphere partition function of any abelian GLSM satisfies a system of differential equations (6.3, 6.4) that are a generalization of the $A$-hypergeometric system defined by Gelfand, Kapranov and Zelevinski (GKZ) [30,31. When the abelian GLSM corresponds to a complete intersection in a toric variety this set of differential equations is precisely the $A$ hypergeometric system. We know from special geometry that the partition function (or $e^{-K}$ ) is 
a symplectic combination of the holomorphic periods and their complex conjugates. The periods themselves must therefore be a solution to this system of differential equations.

Consider a general abelian GLSM with $G=U(1)^{s} \times \Gamma$ and $n$ chiral fields $\Phi_{i=1, \cdots, n}$ with charges $\boldsymbol{Q}_{i}$ and R-charge $\mathrm{q}_{i}$. $\Gamma$ is a discrete abelian gauge group factor that acts on the fields $\Phi_{i}$ through multiplication by a phase $e^{i 2 \pi \varphi_{i}(\gamma)}$, where $\gamma \in \Gamma$ is a group element and $\varphi_{i}(\gamma) \in \mathbb{Q}$. The set $\mathcal{T}$ of $G$-invariant Laurent monomials

$$
\mathcal{T}=\left\{\prod_{i=1}^{n} \phi_{i}^{t_{i}} \mid\left(t_{i}\right) \in \mathbb{Z}^{n}, \sum_{i} t_{i} \boldsymbol{Q}_{i}=0, \sum_{i=1}^{n} \varphi_{i}(\gamma) t_{i} \in \mathbb{Z}\right\},
$$

is parameterized by a lattice that we will denote by $M \cong \mathbb{Z}^{n-s}$, following [49]. This parameterization can be defined in terms of a set of vectors $A:=\left\{v_{1}, \cdots, v_{n}\right\}$ in the dual lattice $N:=\operatorname{Hom}(M, \mathbb{Z})$ as

$$
\mathcal{T}=\left\{\prod_{i=1}^{n} \phi_{i}^{v_{i}(m)} \mid m \in M\right\} .
$$

Let $L:=\left\{\left(l_{i}\right) \in \mathbb{Z}^{n} \mid \sum_{i=1}^{n} l_{i} v_{i}=0\right\}$, denote the lattice of relations amongst the $n$ vectors in $A$. Note that this lattice $L$ is generated over the integers by the vectors $\left\{Q_{i a}\right\}_{a=1,2, \cdots, s}$. For any $\beta \in N \otimes \mathbb{C}$, we define a set of differential equations - which we refer to as the $A$-system - for a function $\Psi\left(x_{1}, \cdots, x_{n}\right)$ :

$$
\begin{gathered}
\sum_{i=1}^{n} v_{i}(m) x_{i} \frac{\partial}{\partial x_{i}} \Psi=\beta(m) \Psi, \quad \forall m \in M, \\
\prod_{l_{i}>0}\left(\frac{\partial}{\partial x_{i}}\right)^{l_{i}} \Psi=\prod_{l_{i}<0}\left(\frac{\partial}{\partial x_{i}}\right)^{\left|l_{i}\right|} \Psi, \quad \forall l \in L .
\end{gathered}
$$

The $A$-system is a slight generalization of the GKZ system since we do not impose the condition (see [30,31]) that $v_{i}$ generate the lattice $N$, or that there should exist an element $\operatorname{deg} \in M$ such that $\operatorname{deg}\left(v_{i}\right)=1$ for $i=1, \cdots, n$.

Our moduli space $\mathcal{M}$ is isomorphic to $\left(\mathbb{C}^{*}\right)^{s}$ and has a natural compactification $\overline{\mathcal{M}}$ specified by the secondary fan of the GLSM. This compactification uses the traditional description of a toric variety in terms of a fan in a real vector space (see, for example, [50] for an exposition of this approach), and the boundary points produced in this way include a point of maximal codimension associated to each GLSM phase around which we can expand physical quantities such as $Z_{S^{2}}$.

A more familiar description of $\overline{\mathcal{M}}$ is as a holomorphic quotient [51, and this construction of it will also allow us to describe the GKZ system. We introduce an auxiliary space $\mathcal{V} \cong \mathbb{C}^{n}$ with coordinates $x_{i=1, \cdots, n}$ along with a $\left(\mathbb{C}^{*}\right)^{n-s}$ action: $x_{i} \sim \lambda_{i}^{v_{i \alpha}} x_{i}$, where $v_{i} \in A$. The variables $x_{i}$ are the homogeneous coordinates of the toric variety $\sqrt{6} \overline{\mathcal{M}}=(\mathcal{V}-\mathcal{Z}) /\left(\mathbb{C}^{*}\right)^{n-s}$ whose fan is the secondary fan corresponding to the abelian GLSM. The FI parameters are specified in terms of the $x_{i}$ as

$$
z_{a}=\prod_{i=1}^{n} x_{i}^{Q_{i a}}
$$

The partition function, which is a real-valued function on the space of FI parameters $\left(\mathbb{C}^{*}\right)^{s}$, can be

\footnotetext{
${ }^{6}$ Here $\mathcal{Z}$ is a closed subset of $\mathcal{V}$ determined by the fan, which must be removed before the quotient is taken. See [50] for an explanation of how this works in detail.
} 
extended to a function $\Psi(x, \bar{x})$ on the space $\mathcal{V}$ as

$$
\begin{aligned}
\Psi(x, \bar{x}) & =\left(\prod_{i=1}^{n}\left(x_{i} \bar{x}_{i}\right)^{-\frac{\mathrm{q}_{i}}{2}}\right) Z_{S^{2}}\left(\prod_{i=1}^{n} x_{i}^{Q_{i a}}, \prod_{i=1}^{n} \bar{x}_{i}^{Q_{i a}}\right) \\
& =\sum_{\boldsymbol{m} \in \mathbb{Z}^{s}} \int \prod_{a=1}^{s} \frac{d \tau_{a}}{2 \pi i} \prod_{i=1}^{n} x_{i}^{-\frac{\mathrm{q}_{i}}{2}+\boldsymbol{Q}_{i} \cdot\left(\tau-\frac{m}{2}\right)} \bar{x}_{i}^{-\frac{\mathrm{q}_{i}}{2}+\boldsymbol{Q}_{i} \cdot\left(\tau+\frac{m}{2}\right)} \frac{\Gamma\left(\frac{\mathrm{q}_{i}}{2}-\boldsymbol{Q}_{i} \cdot\left(\boldsymbol{\tau}+\frac{\boldsymbol{m}}{2}\right)\right)}{\Gamma\left(1-\frac{\mathrm{q}_{i}}{2}+\boldsymbol{Q}_{i} \cdot\left(\boldsymbol{\tau}-\frac{\boldsymbol{m}}{2}\right)\right)}
\end{aligned}
$$

Note that the function $\Psi$ is singular on the compactification $\overline{\mathcal{V}}$ as expected, since the metric on moduli space is singular at the boundaries. Consider a particular phase of the GLSM defined by $\sum_{b=1}^{s} p_{a b} r_{b}>0$, which corresponds to a singular point at $\xi_{a}=0, a=1, \cdots, s$, where $\xi_{a}:=$ $\prod_{b=1}^{s} z_{b}^{p_{a b}}$. The partition function in this phase is a function of the variables $(\xi, \bar{\xi})$. The prefactor in (6.6) can be expressed as $\left|\prod_{a=1}^{s} \xi_{a}^{\delta_{a}}\right|^{2}$, where $\delta_{a} \in \mathbb{Q}$, using the $\left(\mathbb{C}^{*}\right)^{n-s}$ action on $\mathcal{V}$. Therefore, $\Psi$ agrees with $Z_{S^{2}}$ up to a Kähler transformation and hence contains the same physics as the partition function.

Although $x_{i}$ and $\bar{x}_{i}$ are related by complex conjugation we regard them as independent variables. $\Psi(x, \bar{x})$ will therefore be viewed as a holomorphic function in the variables $x_{i}$ and $\bar{x}_{i}$.

Theorem: $\Psi(x, \bar{x})$ satisfies the A-system of differential equations (6.3), (6.4), with $\beta=-\frac{1}{2} \sum_{i=1}^{n} \mathrm{q}_{i} v_{i}$.

For a proof of this assertion see Appendix A. The $A$-system, which $\Psi(x, \bar{x})$ satisfies, is a generalization of the $A$-hypergeometric system defined by GKZ. For some GLSMs the $A$-system is precisely a GKZ system; this includes any abelian GLSM with a geometric phase describing a Calabi-Yau threefold which is a complete intersection in a toric variety (CICY). However, we have shown that one can associate an $A$-system to any GLSM with an abelian gauge group - a broader class of GLSMs than those that describe CICYs. For SCFTs that have integral left and right moving R-charges, $\beta$ is an integral vector since every element of the lattice $\mathcal{T}$ must have even vector R-charge.

As a simple example of an $A$-system which is not of GKZ type, consider the $P^{2}$-GLSM from the previous section. The gauge group is $U(1) \times \mathbb{Z}_{2}$ with chiral fields $X_{1,2,3,4}$ with charge +1 and a field $P$ of charge -4 . The $\mathbb{Z}_{2}$ gauge symmetry acts as $P \rightarrow-P$. The superpotential is given by $W=P^{2} G_{8}(X)$, and we can assign the R-charges $\mathrm{q}_{x}$ to $X_{1,2,3,4}$ and $\mathrm{R}$-charge $1-4 \mathrm{q}_{x}$ to $P$. The lattice of $G$-invariant Laurent monomials can be parameterized in terms of a lattice $M \cong \mathbb{Z}^{4}$ as

$$
\mathcal{T}=\left\{X_{1}^{2 m_{1}+m_{2}} X_{2}^{2 m_{1}+m_{3}} X_{3}^{2 m_{1}+m_{4}} X_{4}^{2 m_{1}-m_{1}-m_{2}-m_{3}} P^{2 m_{1}} \mid m \in M=\mathbb{Z}^{4}\right\},
$$

which determines the set $A=\left\{v_{1}, v_{2}, v_{3}, v_{4}, v_{p}\right\} \subset N \cong \mathbb{Z}^{4}$ as follows:

$$
A=\{(2,1,0,0),(2,0,1,0),(2,0,0,1),(2,-1,-1,-1),(2,0,0,0)\} .
$$

Note that the vectors in $A$ do not generate the lattice $N$, and lie on a hyperplane that has normal distance 2 from the origin $(0,0,0,0)$. The lattice of relations is given by

$$
L=\{(l, l, l, l,-4 l) \mid l \in \mathbb{Z}\},
$$

and the vector $\beta=(-1,0,0,0)$.

In section 5.1 we argued that this GLSM actually describes a Calabi-Yau threefold geometry 
- a special degree 8 hypersurface in $\mathbb{P}^{1,1,1,1,4}$ — in one of its phases. Mirror symmetry provides a description of the quantum Kähler moduli space in this case. The Picard-Fuchs operator is given by [25]

$$
\mathcal{L}_{P F}=(w-256)\left(w \frac{\partial}{\partial w}\right)^{4}+2 w\left(w \frac{\partial}{\partial w}\right)^{3}+\frac{43 w}{32}\left(w \frac{\partial}{\partial w}\right)^{2}+\frac{11 w}{32}\left(w \frac{\partial}{\partial w}\right)+\frac{105 w}{4096}
$$

in terms of an algebraic coordinate $w$ on the moduli space. The $A$-system defined above reduces to a single order 4 linear differential operator in the coordinate $z=\frac{x_{1} x_{2} x_{3} x_{4}}{x_{p}{ }^{4}}$ :

$$
z \prod_{n=0}^{3}\left(-\frac{2 n+1}{2}+z \frac{\partial}{\partial z}\right)-\left(z \frac{\partial}{\partial z}\right)^{4}
$$

It is easily checked that this differential operator agrees with the Picard-Fuchs operator (6.10) derived using mirror symmetry, if the coordinates $z$ and $w$ are related as $w=\frac{1}{256 z^{4}}$.

As in our discussion of phases, the associated Cartan theory provides some information about the periods of a general non-abelian GLSM. The associated Cartan theory, being an abelian GLSM, can be associated with an $A$-system. This system of differential equations can be solved in a given GLSM phase yielding a complete set of solutions. On restriction to the Weyl-invariant subspace of the FI parameter space, we expect the periods of the non-abelian theory to be contained in the space of solutions. We postpone a more detailed study of the non-abelian GLSM along these lines to future work.

\section{Conclusions}

In this paper we have utilized the two-sphere partition function $Z_{S^{2}}$ to study three aspects of GLSM physics: the appearance of classical phase transitions upon movement in the space of FI parameters, criteria necessary for the existence of a geometric phase, and a system of differential equations on the moduli space of the IR SCFT.

We saw in section 3 that the analytic structure of the partition function integrand recovers the description of abelian GLSM phases in terms of the secondary fan [33 35]. Passing through codimension one walls in FI space moves the theory to a different maximal cone of the secondary fan; this requires a different choice of contours in the partition function integral, and the phase transition is detected through a change in the structure of residues. Some non-abelian phase transitions can be detected by studying the secondary fan of the associated Cartan theory, as demonstrated in the Gulliksen-Negård GLSM.

In section 4 we developed a set of geometric phase criteria, which a GLSM phase must satisfy in order to admit a description in terms of a Calabi-Yau non-linear sigma model. These criteria, which include conditions on the partition function, can be easily checked in a given GLSM phase. We formulated another potential criterion and demonstrated that its violation suggests the existence of singularities in the geometry, using hypersurfaces in weighted projective space as an example. We also constructed a GLSM which has a phase satisfying all the criteria, but is actually singular due to the existence of non-compact directions in the moduli space of vacua. This shows that the geometric phase criteria are necessary but not sufficient for the existence of a geometric phase.

In section 5 we applied the geometric phase criteria to identify a set of novel geometric phases 
in GLSMs. We studied a collection of GLSMs with gauge group $U(1)$ that satisfy the criteria. Focusing on one member of the collection, we showed that the relevant phase looks like a hybrid: two copies of $\mathbb{P}^{3}$ glued together by a Landau-Ginzburg model. We then realized the same phase in an auxiliary two-parameter GLSM and showed that the hybrid phase is actually geometric, using the following physical argument - the IR physics in the auxiliary GLSM is the same along codimension-one loci in FI space, and one such locus connects the hybrid phase of interest to a geometric phase. The Calabi-Yau threefold realized by the hybrid phase is a double-cover of $\mathbb{P}^{3}$ branched over an octic surface.

In section 6 we demonstrated that a system of linear differential equations, which we call the $A$-system, can be associated to any abelian GLSM, and $Z_{S^{2}}$ is a solution of this system. For abelian GLSMs which describe complete intersections in a toric variety, this system reduces to the $A$-hypergeometric system of Gel'fand, Kapranov and Zelevinski. We leave an investigation along these lines for non-abelian theories to future work.

Another interesting direction for future work is to apply the method of section 5 to large collections of GLSMs specified by the gauge group, matter representations and R-charges. The geometric phase criteria would then give us a set of candidate Calabi-Yau threefold geometries. Upon closer study, some of these GLSMs could lead to novel constructions of Calabi-Yau threefolds.

Acknowledgments. We thank F. Benini, M. Cvetič, R. Donagi, J. Gomis, R. Gopakumar, A. Grassi, K. Hori, S. Hosono, N. Iqbal, H. Jockers, D. Klevers, J. M. Lapan, B. Le Floch, S. Lee, L. McAllister, R. Plesser, M. Romo, A. Sen, and S. Trivedi for useful discussions and S. K. Harris and J. M. Lapan for comments on a draft. We are especially grateful to J. M. Lapan for extensive discussions on the $P^{2}$-GLSM and related issues. V. K. would like to thank the Tata Institute for Fundamental Research, the Harish-Chandra Research Institute, for hospitality while this research was being carried out, and the participants of the International Strings Meeting 2012, Puri, India for interesting discussions. V. K. and D. R. M. are grateful to the organizers and participants of the workshop "Seminar weeks on Calabi-Yau manifolds, mirror symmetry, and derived categories" at the University of Tokyo for useful discussions, and D. R. M. is equally grateful to the organizers and participants of the meeting "New Mathematical Structures in Supersymmetric Gauge Theory?" at the Perimeter Institute for Theoretical Physics. The work of J. H. and V. K. was supported in part by the National Science Foundation under Grant No. PHY11-25915, and the work of D. R. M. was supported in part by National Science Foundation Grant DMS-1007414.

\section{A GKZ Proof}

The property $\sum_{i=1}^{n} \boldsymbol{Q}_{i} v_{i}=0$ is sufficient to demonstrate that (6.3) is satisfied.

We now show that $\Psi(x, \bar{x})$ as defined in (6.6) is a solution to the set of differential operators (6.4). In any given phase of the GLSM the $s$-dimensional contour integral can be expressed as a sum of residues at points that lie inside the contour of integration. The integral naturally takes the form of an infinite power series, and we will verify equation (6.4) term wise by comparing coefficients.

Consider the contribution to the integral (6.6) from a single pole located at the intersection of

$s$ pole hyperplanes $H_{\alpha}^{\left(k_{\alpha}\right)}$, for $\alpha \in I$, where $I \subset\{1, \cdots, n\}$ is an s-element subset, with $\left\{\boldsymbol{Q}_{\alpha}\right\}_{\alpha \in I}$ linearly independent. For each $\boldsymbol{m} \in \mathbb{Z}^{s}$, we study the contribution from the pole located at 
$\boldsymbol{\tau}=-\boldsymbol{m} / 2+\boldsymbol{\tau}_{\boldsymbol{k}}$, which is the unique solution of the equations

$$
\frac{\mathrm{q}_{\alpha}}{2}-\boldsymbol{Q}_{\alpha} \cdot\left(\boldsymbol{\tau}+\frac{\boldsymbol{m}}{2}\right)=-k_{\alpha}, \quad \text { for } \alpha \in I .
$$

The residue contribution, for a fixed $\boldsymbol{m} \in \mathbb{Z}^{s}$, is

$$
\Psi_{\boldsymbol{k}, \boldsymbol{m}}=\operatorname{Res}_{\boldsymbol{\epsilon}=0} \prod_{i=1}^{n} x_{i}^{-\frac{\mathrm{q}_{i}}{2}+\boldsymbol{Q}_{i} \cdot\left(\boldsymbol{\tau}_{\boldsymbol{k}}+\boldsymbol{\epsilon}-\boldsymbol{m}\right)} \bar{x}_{i}^{-\frac{\mathrm{q}_{i}}{2}+\boldsymbol{Q}_{i} \cdot\left(\boldsymbol{\tau}_{\boldsymbol{k}}+\boldsymbol{\epsilon}\right)} \frac{\Gamma\left(\frac{\mathrm{q}_{i}}{2}-\boldsymbol{Q}_{i} \cdot\left(\boldsymbol{\tau}_{\boldsymbol{k}}+\boldsymbol{\epsilon}\right)\right)}{\Gamma\left(1-\frac{\mathrm{q}_{i}}{2}+\boldsymbol{Q}_{i} \cdot\left(\boldsymbol{\tau}_{\boldsymbol{k}}+\boldsymbol{\epsilon}-\boldsymbol{m}\right)\right)} .
$$

Since the lattice $L$ is generated by the vectors $Q_{i, a=1}, Q_{i, a=2}, \cdots, Q_{i, a=s}$, we can express $l_{i}=Q_{i a} p_{a}$, where $p_{a} \in \mathbb{Z}$. Using standard $\Gamma$-function identities we have

$$
\begin{aligned}
\prod_{l_{i}>0}\left(\frac{\partial}{\partial x_{i}}\right)^{l_{i}} \Psi_{\boldsymbol{k}, \boldsymbol{m}}= & \operatorname{Res}_{\boldsymbol{\epsilon}=0} \prod_{i=1}^{n} x_{i}^{-\frac{\mathrm{q}_{i}}{2}+\boldsymbol{Q}_{i} \cdot\left(\boldsymbol{\tau}_{\boldsymbol{k}}+\boldsymbol{\epsilon}-\boldsymbol{m}\right)} \bar{x}_{i}^{-\frac{\mathrm{q}_{i}}{2}+\boldsymbol{Q}_{i} \cdot\left(\boldsymbol{\tau}_{\boldsymbol{k}}+\boldsymbol{\epsilon}\right)} \frac{\Gamma\left(\frac{\mathrm{q}_{i}}{2}-\boldsymbol{Q}_{i} \cdot\left(\boldsymbol{\tau}_{\boldsymbol{k}}+\boldsymbol{\epsilon}\right)\right)}{\Gamma\left(1-\frac{\mathrm{q}_{i}}{2}+\boldsymbol{Q}_{i} \cdot\left(\boldsymbol{\tau}_{\boldsymbol{k}}+\boldsymbol{\epsilon}-\boldsymbol{m}\right)\right)} \\
& \prod_{l_{j}>0} \frac{1}{x_{j}^{l_{j}}} \frac{\Gamma\left(1-\frac{\mathrm{q}_{j}}{2}+\boldsymbol{Q}_{j} \cdot\left(\boldsymbol{\tau}_{\boldsymbol{k}}+\boldsymbol{\epsilon}-\boldsymbol{m}\right)\right)}{\Gamma\left(1-\frac{\mathrm{q}_{j}}{2}+\boldsymbol{Q}_{j} \cdot\left(\boldsymbol{\tau}_{\boldsymbol{k}}+\boldsymbol{\epsilon}-\boldsymbol{m}\right)-l_{j}\right)} \\
= & \operatorname{Res}_{\boldsymbol{\epsilon}=0}^{n} \prod_{i=1}^{n} x_{i}^{-\frac{\mathrm{q}_{i}}{2}+\boldsymbol{Q}_{i} \cdot\left(\boldsymbol{\tau}_{\boldsymbol{k}}+\boldsymbol{\epsilon}-\boldsymbol{m}\right)-l_{i}} \bar{x}_{i}^{-\frac{\mathrm{q}_{i}}{2}+\boldsymbol{Q}_{i} \cdot\left(\boldsymbol{\tau}_{\boldsymbol{k}}+\boldsymbol{\epsilon}\right)} \frac{\Gamma\left(\frac{\mathrm{q}_{i}}{2}-\boldsymbol{Q}_{i} \cdot\left(\boldsymbol{\tau}_{\boldsymbol{k}}+\boldsymbol{\epsilon}\right)\right)}{\Gamma\left(1-\frac{\mathrm{q}_{i}}{2}+\boldsymbol{Q}_{i} \cdot\left(\boldsymbol{\tau}_{\boldsymbol{k}}+\boldsymbol{\epsilon}-\boldsymbol{m}\right)-l_{i}\right)} \\
& \prod_{l_{j}<0} \frac{1}{x_{j}^{\left|l_{j}\right|}} \frac{\Gamma\left(1-\frac{\mathrm{q}_{j}}{2}+\boldsymbol{Q}_{j} \cdot\left(\boldsymbol{\tau}_{\boldsymbol{k}}+\boldsymbol{\epsilon}-\boldsymbol{m}\right)-l_{j}\right)}{\Gamma\left(1-\frac{\mathrm{q}_{j}}{2}+\boldsymbol{Q}_{j} \cdot\left(\boldsymbol{\tau}_{\boldsymbol{k}}+\boldsymbol{\epsilon}-\boldsymbol{m}\right)\right)} \\
= & \prod_{l_{i}<0}\left(\frac{\partial}{\partial x_{i}}\right)^{\left|l_{i}\right|} \Psi_{\boldsymbol{k}, \boldsymbol{m}+\boldsymbol{p}}
\end{aligned}
$$

Now, the contribution to $\Psi$ from the pole located at $\boldsymbol{\tau}=-\boldsymbol{m} / 2+\boldsymbol{\tau}_{\boldsymbol{k}}$ is obtained by summing over all $\boldsymbol{m} \in \mathbb{Z}^{s}$, and then summing over all contributing poles. Through a relabeling $\boldsymbol{m} \rightarrow \boldsymbol{m}-\boldsymbol{p}$, it is easily seen that

$$
\prod_{l_{i}>0}\left(\frac{\partial}{\partial x_{i}}\right)^{l_{i}} \sum_{\text {poles }} \sum_{\boldsymbol{m} \in \mathbb{Z}^{s}} \Psi_{\boldsymbol{k}, \boldsymbol{m}}=\prod_{l_{i}<0}\left(\frac{\partial}{\partial x_{i}}\right)^{\left|l_{i}\right|} \sum_{\text {poles }} \sum_{\boldsymbol{m} \in \mathbb{Z}^{s}} \Psi_{\boldsymbol{k}, \boldsymbol{m}}
$$

Therefore, we see that equation (6.4) is true in any phase of the GLSM.

\section{B Evaluation of the Partition Function}

The discussion of section 4 discussed contributions to $Z_{S^{2}}$ from point sets $P_{I}$. At a given point $\boldsymbol{r}$ in the space of FI-parameters, there may be many such sets, and an important practical consideration is how to set up sums in the partition function which ensure that all contributing points are identified. Though there are likely better methods, a simple method is to set up a "grid."

To illustrate the idea, consider a simple example. Take the $\mathbb{P}_{1,1,2,2,2}^{4}$ GLSM, which has two fields $\phi_{i}$ with $Q_{i}=1$ and $\mathrm{q}_{i}=0$, three fields $\chi_{i}$ with $Q_{i}=2$ and $\mathrm{q}_{i}=0$, and one field $P$ with $Q_{i}=-8$ 
and $\mathrm{q}_{i}=2$. The one-loop determinants are given by

$$
Z_{\phi}=\frac{\Gamma\left(-\tau-\frac{m}{2}\right)}{\Gamma\left(1+\tau-\frac{m}{2}\right)} \quad Z_{\chi}=\frac{\Gamma(-2 \tau-m)}{\Gamma(1+2 \tau-m)} \quad Z_{P}=\frac{\Gamma(1+8 \tau+4 m)}{\Gamma(-8 \tau+4 m)} .
$$

The $\Gamma$-functions in the numerators define infinite sets of polar hyperplanes where the arguments are negative integers. The points $p$ in $\tau$-space which are in the intersection of many such hyperplanes have $\tau_{a} \in \boldsymbol{Q}$ for all $a$, and for simplicity we set up a summation which contains all contributing points $p$ in all phases. However, this requires a careful choice of summation variable: if one were to sum over $\tau=k-\frac{m}{2}$ with $k \in \mathbb{Z}$, all $Z_{\phi}$ poles but only every second $Z_{\chi}$ pole and every eighth $Z_{P}$ pole would be in the sum. Instead, if one sums over $\tau=\frac{k}{n}-\frac{m}{2}$ with $k \in \mathbb{Z}$ and $n=8$ every pole would be in the sum. More generically, a similar expansion holds for any $\mathbb{W P}^{4}$ GLSM with $n=\operatorname{lcm}(F), F=\left\{Q_{i}\right\}$.

It is easy to see how the one-dimensional grid just described generalizes to a grid which captures all contributing points $p$ for any GLSM 7 . Defining the sets of charges $F_{a}=\left\{Q_{i a}\right\}$ and $n_{a}=l c m\left(F_{a}\right)$, sum over $\tau_{a}=\frac{k_{a}}{n_{a} K}-\frac{m_{a}}{2}$ with $k_{a} \in \mathbb{Z}$, where $K$ depends on the R-charges. For example, for any GLSM with integer R-charges $\mathrm{q}_{i}, K=2$ suffices. Having such a sum over poles, one integrates in neighborhoods of the poles defined by $\tau_{a}+\epsilon_{a}=\frac{k_{a}}{n_{a} K}-\frac{m_{a}}{2}+\epsilon_{a}$ and the integrals take the form $\oint \ldots \oint \frac{d \epsilon_{1}}{2 \pi i} \cdots \frac{d \epsilon_{s}}{2 \pi i}$. Rewriting $Z_{\text {class }}$ in terms of these new variables, and also replacing $m_{a}$ via defining a new variable $l_{a}:=\frac{k_{a}}{n_{a} K}-m_{a}$, we obtain

$$
Z_{\text {class }}=e^{-4 \pi \boldsymbol{r} \cdot \boldsymbol{\tau}-i \boldsymbol{\theta} \cdot \boldsymbol{m}}=\prod_{a}\left(z_{a} \bar{z}_{a}\right)^{\epsilon_{a}} z_{a}^{l_{a}} \bar{z}_{a}^{\frac{k_{a}}{n_{a} K}} .
$$

Note that unless $k_{a}=0 \bmod \left(n_{a} K\right) \quad \forall a$, some number of the $z_{a}$ variables will be fractional. If there are non-trivial poles for such $k_{a}$, the fractional powers appear in the partition function $Z_{S^{2}}$, and in some examples we will see that fractional powers are related to singular geometries 4.3. Note also that two distinct sets of $k_{a}$ 's which are equivalent $\bmod \left(n_{a} K\right)$ for all $a$ define points in $\tau_{a}$ space with the same structure of polar divisors. This defines a "modular box". Finally, since the summation variables $k_{a}$ have been set up in a phase independent manner, the choice of a phase generically requires that the $k_{a}$ satisfy certain inequalities governed by the choice of contours. This is simple to address on an example by example basis.

\section{References}

[1] E. Witten, "Phases of $\mathcal{N}=2$ Theories in Two-Dimensions," Nucl.Phys. B403 (1993) 159-222, hep-th/9301042.

[2] F. Benini and S. Cremonesi, "Partition Functions of $\mathrm{N}=(2,2)$ Gauge Theories on $\mathrm{S}^{2}$ and Vortices," 1206.2356.

[3] N. Doroud, J. Gomis, B. Le Floch, and S. Lee, "Exact Results in D=2 Supersymmetric Gauge Theories," 1206.2606.

[4] H. Jockers, V. Kumar, J. M. Lapan, D. R. Morrison, and M. Romo, "Two-Sphere Partition Functions and Gromov-Witten Invariants," 1208.6244.

\footnotetext{
${ }^{7}$ Both abelian and non-abelian since the poles of $Z_{S^{2}}^{N A}$ are a subset of $Z_{S^{2}}^{C}$ poles.
} 
[5] J. Gomis and S. Lee, "Exact Kahler Potential from Gauge Theory and Mirror Symmetry," JHEP 1304 (2013) 019, 1210.6022.

[6] Y. Honma and M. Manabe, "Exact Kahler Potential for Calabi-Yau Fourfolds," arXiv:1302.3760 [hep-th].

[7] D. S. Park and J. Song, "The Seiberg-Witten Kahler Potential as a Two-Sphere Partition Function," 1211.0019.

[8] E. Sharpe, "Predictions for Gromov-Witten invariants of noncommutative resolutions," 1212.5322 .

[9] K. Hori and C. Vafa, "Mirror Symmetry," hep-th/0002222.

[10] I. Ciocan-Fontanine, B. Kim, and C. Sabbah, "The Abelian/Nonabelian Correspondence and Frobenius Manifolds," Invent. Math. 171 (2008), no. 2, 301-343, math/0610265.

[11] K. Hori and D. Tong, "Aspects of Non-Abelian Gauge Dynamics in Two-Dimensional $\mathrm{N}=(2,2)$ Theories," JHEP 0705 (2007) 079, hep-th/0609032.

[12] P. S. Aspinwall and B. R. Greene, "On the geometric interpretation of $\mathrm{N}=2$ superconformal theories," Nucl.Phys. B437 (1995) 205-230, hep-th/9409110.

[13] A. Căldăraru, J. Distler, S. Hellerman, T. Pantev, and E. Sharpe, "Non-birational twisted derived equivalences in abelian GLSMs," Comm. Math. Phys. 294 (2010), no. 3, 605-645, arXiv:0709.3855 [hep-th].

[14] S. Hellerman, A. Henriques, T. Pantev, E. Sharpe, and M. Ando, "Cluster decomposition, T-duality, and gerby CFTs," Adv. Theor. Math. Phys. 11 (2007), no. 5, 751-818, arXiv:hep-th/0606034.

[15] E. Sharpe, "Predictions for Gromov-Witten invariants of noncommutative resolutions," 1212.5322.

[16] B. de Wit and A. Van Proeyen, "Potentials and Symmetries of General Gauged N=2 Supergravity: Yang-Mills Models," Nucl. Phys. B 245 (1984) 89-117.

[17] V. Periwal and A. Strominger, "Kähler geometry of the space of N=2 superconformal field theories," Phys. Lett. B 235 (1990) 261.

[18] A. Strominger, "Special Geometry," Commun. Math. Phys. 133 (1990) 163-180.

[19] P. Candelas and X. de la Ossa, "Moduli Space of Calabi-Yau Manifolds," Nucl.Phys. B355 (1991) 455-481.

[20] B. Craps, F. Roose, W. Troost, and A. Van Proeyen, "What is special Kähler geometry?," Nucl. Phys. B 503 (1997) 565-613, hep-th/9703082.

[21] R. L. Bryant and P. A. Griffiths, "Some observations on the infinitesimal period relations for regular threefolds with trivial canonical bundle," in Arithmetic and geometry, Vol. II, vol. 36 of Progr. Math., pp. 77-102. Birkhäuser Boston, Boston, MA, 1983.

[22] D. S. Freed, "Special Kähler manifolds," Commun. Math. Phys. 203 (1999) 31-52, hep-th/9712042.

[23] A. C. Cadavid and S. Ferrara, "Picard-Fuchs equations and the moduli space of superconformal field theories," Phys. Lett. B267 (1991) 193-199.

[24] W. Lerche, D. J. Smit, and N. P. Warner, "Differential equations for periods and flat coordinates in two-dimensional topological matter theories," Nucl. Phys. B372 (1992) 87-112, hep-th/9108013. 
[25] D. R. Morrison, "Picard-Fuchs Equations and Mirror Maps for Hypersurfaces," in Essays on Mirror Manifolds, S.-T. Yau, ed., pp. 241-264. International Press, Hong Kong, 1992. hep-th/9111025.

[26] P. Deligne, Équations différentielles à points singuliers réguliers. Springer-Verlag, Berlin, 1970. Lecture Notes in Mathematics, Vol. 163.

[27] V. V. Batyrev, "Variations of the Mixed Hodge Structure of Affine Hypersurfaces in Algebraic Tori," Duke Math. J. 69 (1993) 349-409.

[28] V. V. Batyrev and D. van Straten, "Generalized hypergeometric functions and rational curves on Calabi-Yau complete intersections in toric varieties," Comm. Math. Phys. 168 (1995), no. 3, 493-533, alg-geom/9307010.

[29] S. Hosono, A. Klemm, S. Theisen, and S.-T. Yau, "Mirror Symmetry, Mirror Map and Applications to Calabi-Yau Hypersurfaces," Commun.Math.Phys. 167 (1995) 301-350, hep-th/9308122.

[30] I. Gelfand, M. Kapranov, and A. Zelevinsky, "Hypergeometric Functions and Toric Varieties," Funktsional. Anal. 23 (1989) 12 - 26.

[31] I. Gelfand, M. Kapranov, and A. Zelevinsky, "Generalized Euler integrals and A-hypergeometric functions," Advances in Mathematics 84 (1990), no. 2, 255 - 271.

[32] E. Silverstein and E. Witten, "Global U(1) R symmetry and conformal invariance of $(0,2)$ models," Phys.Lett. B328 (1994) 307-311, hep-th/9403054.

[33] I. M. Gel'fand, A. V. Zelevinskiü, and M. M. Kapranov, "Newton polyhedra of principal A-determinants," Soviet Math. Dokl. 40 (1990) 278-281.

[34] L. J. Billera, P. Filliman, and B. Sturmfels, "Constructions and complexity of secondary polytopes," Adv. Math. 83 (1990), no. 2, 155-179.

[35] P. S. Aspinwall, B. R. Greene, and D. R. Morrison, "Calabi-Yau Moduli Space, Mirror Manifolds and Space-Time Topology Change in String Theory," Nucl.Phys. B416 (1994) 414-480, hep-th/9309097.

[36] P. Griffiths and J. Harris, Principles of algebraic geometry. John Wiley \& Sons, New York, 1978.

[37] R. P. Horja, "Hypergeometric functions and mirror symmetry in toric varieties," arXiv:math.AG/9912109,

[38] J. Stienstra, "GKZ Hypergeometric Structures," math/0511351.

[39] R. N. Cahn, Semi-simple Lie algebras and their representations. Benjamin/cummings Menlo Park, California, 1984.

[40] H. Jockers, V. Kumar, J. M. Lapan, D. R. Morrison, and M. Romo, "Nonabelian 2D Gauge Theories for Determinantal Calabi-Yau Varieties," JHEP 1211 (2012) 166, 1205.3192.

[41] W. Lerche, C. Vafa, and N. P. Warner, "Chiral Rings in N=2 Superconformal Theories," Nucl.Phys. B324 (1989) 427.

[42] A. Sen, "(2, 0) Supersymmetry and Space-Time Supersymmetry in the Heterotic String Theory," Nucl.Phys. B278 (1986) 289.

[43] N. Seiberg, "Observations on the Moduli Space of Superconformal Field Theories," Nucl.Phys. B303 (1988) 286. 
[44] D. R. Morrison, "Mirror Symmetry and Rational Curves on Quintic Threefolds: A Guide For Mathematicians," J. Amer. Math. Soc. 6 (1993) 223-247, arXiv:alg-geom/9202004.

[45] D. R. Morrison, "Compactifications of Moduli Spaces Inspired by Mirror Symmetry," in Journées de Géométrie Algébrique d'Orsay (Juillet 1992), vol. 218 of Astérisque, pp. 243-271. Société Mathématique de France, 1993. arXiv:alg-geom/9304007.

[46] P. Candelas, X. de la Ossa, A. Font, S. H. Katz, and D. R. Morrison, "Mirror Symmetry for Two Parameter Models. I," Nucl. Phys. B416 (1994) 481-538, hep-th/9308083.

[47] P. Candelas, A. Font, S. H. Katz, and D. R. Morrison, "Mirror Symmetry for Two Parameter Models. 2.," Nucl.Phys. B429 (1994) 626-674, hep-th/9403187.

[48] P. S. Aspinwall and M. R. Plesser, "Decompactifications and Massless D-Branes in Hybrid Models," JHEP 1007 (2010) 078, 0909.0252.

[49] D. R. Morrison and M. R. Plesser, "Summing the Instantons: Quantum Cohomology and Mirror Symmetry in Toric Varieties," Nucl. Phys. B 440 (1995) 279-354, arXiv:hep-th/9412236.

[50] P. S. Aspinwall, B. R. Greene, and D. R. Morrison, "Calabi-Yau Moduli Space, Mirror Manifolds and Spacetime Topology Change in String Theory," Nucl. Phys. B 416 (1994) 414-480, arXiv:hep-th/9309097.

[51] D. A. Cox, "The homogeneous coordinate ring of a toric variety," J. Algebraic Geom. 4 (1995) 17-50, arXiv:alg-geom/9210008. 